\title{
THE INVESTIGATION OF FLUID FLOW IN CARTILAGE
}

\section{CONTACT GAP}

JinJing Liao ${ }^{a}$, David W. Smith ${ }^{b}$, Saeed Miraminia ${ }^{a}$ Namal Thibbotuwawad, Bruce S.

\author{
Gardiner $^{\mathrm{c}}$, Lihai Zhang ${ }^{\mathrm{a}^{*}}$
}

a Department of Infrastructure Engineering, The University of Melbourne, Victoria 3010, Australia

b Faculty of Engineering, Computing and Mathematics, The University of Western Australia, WA 6009, Australia

c School of Engineering and Information Technology, Murdoch University, WA 6150, Australia

d School of Chemistry, Physics and Mechanical Engineering, Queensland University of Technology, QLD 4001, Australia

*Corresponding author (Email: lihzhang@unimelb.edu.au)

\section{Highlights:}

- $\quad$ Study fluid flow in cartilage gap by developing validated numerical models.

- $\quad$ Synthesise cartilage topography using the measurements from Dektak Stylus Profilers.

- $\quad$ Contact gap height and fluid pressure gradient greatly affect the gap permeability.

- The gap is functionally closed at particular gap height and pressure gradient. 
Abstract. Synovial fluid flow in articular joint capsule plays an important role during mixed mode lubrication. However, the actual fluid flow behaviour during cartilage contact has not been fully understood so far. This is due to the difficulties in measuring the gap permeability using conventional experimental techniques. The problem becomes further complicated with consideration of the cartilage surface roughness. Here a validated numerical study was developed to quantify the gap permeability to lateral synovial fluid flow. Both macro and micro scale gap flow models were created based on Darcy's law at the macroscale and the Navierstokes equation at the microscale. To generate model inputs, the cartilage topography was numerically synthesised based on the experimental measurements

Keywords: cartilage; contact gap; gap permeability; surface roughness; synovial fluid. of bovine media tibia cartilage using Dektak Stylus Profilers. The experimental results show that the average surface roughness $R_{a}$ is $1.97 \mu m$ and root-mean-square roughness $R_{q}$ is $2.44 \mu \mathrm{m}$, while the correlation lengths of the secondary and tertiary undulations are round $100 \mu \mathrm{m}$ and $20 \mu \mathrm{m}$, respectively. The numerical results indicate that the contact gap height and fluid pressure gradient are two critical parameters which significantly affect the gap permeability. As the contact gap closes, there is a decrease in gap permeability, and most importantly, the gap permeability is also very sensitive to the fluid pressure gradient. Furthermore, with gap closure, the permeability of the contact gap gradually approaches that of the cartilage tissue, at which point the contact gap is functional closed. This occurs at a contact gap around $1 \mu \mathrm{m}$ and for a fluid pressure gradient is below $5 \times 10^{5} \mathrm{~Pa} / \mathrm{m}$. 


\section{INTRODUCTION}

Man-made contacts (e.g. bearings) are designed to be smooth for achieving a low friction (and wear) surface. Animal joint bearings have developed their own solution. Within articulating joints, the porous cartilage surface is relatively rough (see Fig.1), but despite the surface roughness, cartilage's initial friction coefficient of $\sim 0.01$ outperforms any current man-made bearings (Merkher et al., 2006).

Cartilage lubrication and/or frictional properties has been previously organised

by stages of contact between two opposing surfaces, namely hydrodynamic lubrication, mixed lubrication and boundary lubrication (Neu et al., 2008). Mixed mode lubrication which is a state involving hydrodynamic lubrication, boundary lubrication and biphasic lubrication by interstitial fluid pressurisation is responsible for the low friction and wear of cartilage (Ateshian, 2009).

The concept of "weeping lubrication" was first proposed by McCutchen (1959). According to this theory (McCutchen, 1962), the compression of asperities in the contact gap pressurises interstitial fluid within the cartilage, and the interstitial fluid then flows down its pressure gradient to "weep" into the contact gap. With the pressurised incompressible fluid in the contact gap initially carrying most of the applied load, the friction is reduced significantly. This hydrodynamic lubrication mechanism is significantly extended in time due to the fluid lost from the contact gap being replenished by fluid that "weeps" into the contact gap from the cartilage. On the other hand, the boosted lubrication theory was proposed by Walker et al. (1968, 1969), which argued that the pressurised synovial fluid is more likely to undergo ultrafiltration, the gap fluid flowing down its pressure gradient into the cartilage, leaving behind a concentrated protein gel that acted as a boundary lubricant. 
In the last decades, significant progress has been made in the understanding of

biotribology of cartilage. Biphasic lubrication that emphasises on the load support capability of the pressurised interstitial fluid inside cartilages rather than the lubricants in the gap have been proposed (Ateshian, 2009). It is argued that the high-level interstitial fluid pressurisation inside the cartilage could minimise direct solid phase shear between the contact surfaces and hence reduce the friction. Recently, the replenishment of interstitial fluid from imbibition during joint cartilage swell ("tribological rehydration") has been reported by Moore and Burris (2017). Through multiple start-stop cartilage-on-glass sliding tests, they concluded that the interstitial fluid lost in static loading could be recovered during sliding because the hydrodynamic pressure pushed the fluid in the contact gap into cartilage tissue, and the resultant flow into the tissue enhanced solute transport (Graham et al., 2017).

As per the short review above, the fluid flow in the contact gap plays an important role in cartilage mixed mode contact, and hence a better understanding on the fluid flow behaviour in the contact gap is essential. As shown in Fig.1, there are two potential fluid paths from the contact gap, one is through fluid exchange with the cartilage, the other is lateral outflow across the perimeter of the contact gap. The dominance of one path over the other is likely to vary throughout the time course of the contact. Indeed, given the time-dependent poroelastic properties of cartilage, the flow paths and cartilage's frictional properties are expected to change (Zhang et al., 2015). Wu and Ferguson (2017) have laid a foundation on this issue by studying the effects of asperity distribution, root-mean-square (RMS) roughness, wavelengths and flow angle on the contact gap permeability. However as discussed later, in Wu and Ferguson (2017)'s analysis the synovial fluid is assumed to be 
99 Newtonian viscosity, while the randomness of surface roughness is not guaranteed statistically (i.e., sample dimensions and sizes were not large enough, the wavelengths were not random).

Since no contact model has addressed the interaction between the gap flow with non-Newtonian properties (Mow et al., 1993). This study represents a first step towards understanding the fundamental fluid flow behaviour in the contact gap. This understanding is essential not only for understanding synovial joint behaviour, but employed to investigate the fluid flow in contact gap between two cartilages. Finally, also for the development of bio-inspired smart materials (Greene et al., 2014), which have a variety of practical applications in the fields of daily life, industry, and agriculture (e.g. cartilage-inspired lubrication system). We first measured the surface roughness by using bovine cartilage, and then the synthetic surfaces of cartilage were developed by employing an advanced mathematical algorithm with key measured parameters (i.e., average roughness $R_{\mathrm{a}}$, RMS roughness $R_{\mathrm{q}}$ and correlation length). The methodology allows for multiple sample investigations to satisfy statistical requirements with less computational cost. A micro-scale of computational fluid dynamics (CFD) model with non-Newtonian viscosity was the gap permeability can be estimated by the gap height and fluid pressure gradient. 


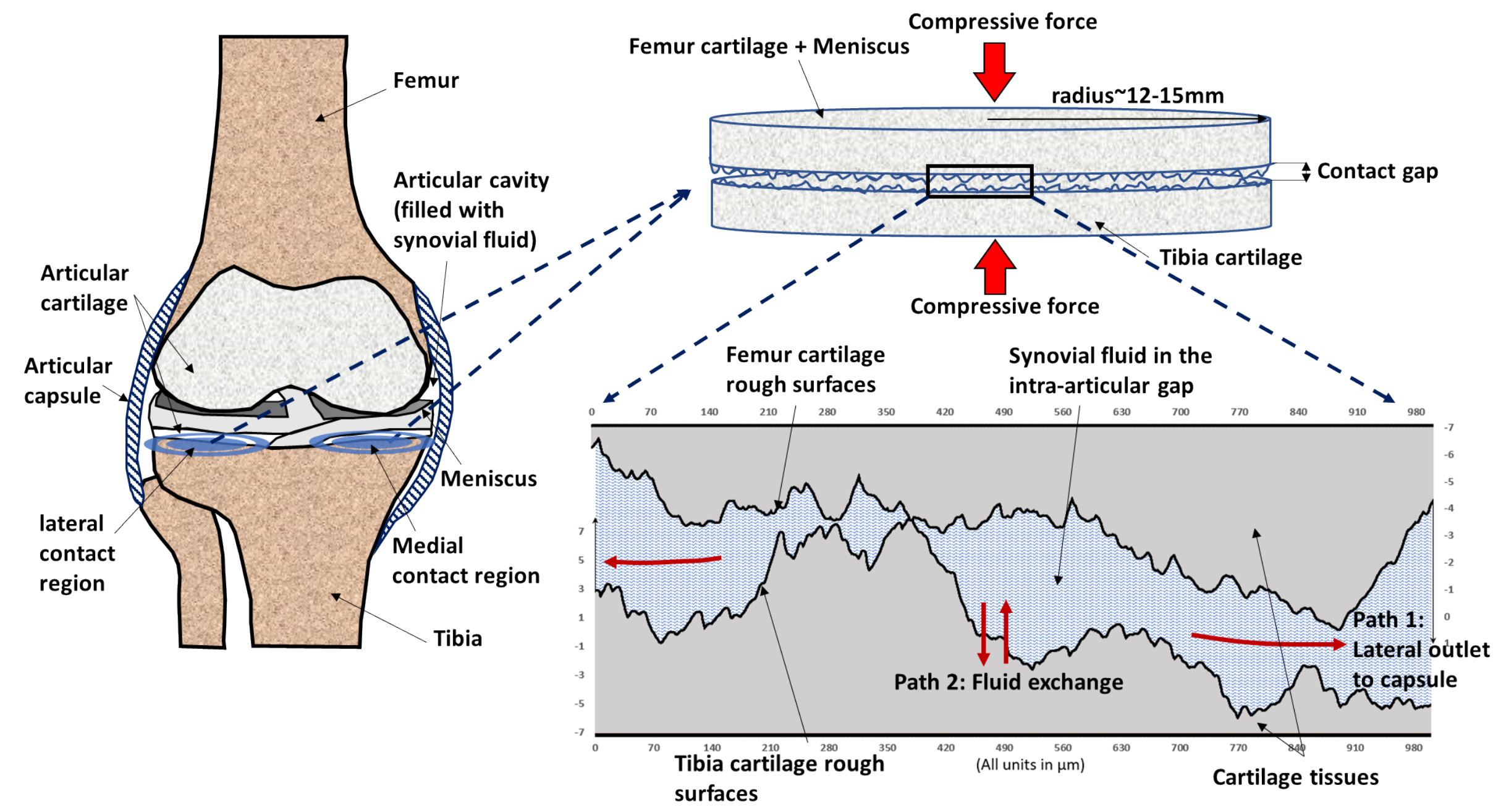

Fig.1 -Schematic diagram showing fluid flow in cartilage contact gap. 


\section{MATERIALS AND METHODS}

\subsection{Methods}

The physical configuration of the problem is a simplification of the synovial knee joint (see Fig.1). By treating the femur and meniscus as one body, we can obtain two approximately circular contact regions between femur-meniscus and tibia (lateral and medial), with a radius of 12-15 $\mathrm{mm}$ each (Marzo and Gurske-DePerio, 2009). Furthermore, by ignoring the femur head curvature, the problem can be simplified as two cylindrical cartilage discs. The above simplifications have often been used when investigating the squeeze film behaviour of joint lubrication (Bujurke and Kudenatti, 2006), in which a thick fluid film separates two cartilages. On initial contact of cartilage surface asperities, the joint lubrication mode shifts from squeeze film to mixed mode lubrication. This general account of gap behaviour is elementary: it simply says that initially the two contacting surface are (potentially) separated by fluid, and then they contact at asperities, establishing simultaneous boundary lubrication and hydrodynamic lubrication, which is referred to as mixed mode lubrication. Based on the measurements in this study, the initial cartilage surface roughness is randomly distributed. A schematic depiction of the inferred cross-section profiles (based on measurement data of the initial profiles) of femur and tibia some time after initial under contact is shown in Fig.1. With the heights and uneven contact of the randomly distributed asperities, there will form an interconnected pore space that is described here as the "contact gap". For a single load event such as standing up for some time, the two cartilage discs can be assumed under quasi-static compression, which will cause the contact gap closure and thereby change the fluid behaviour in the gap.

This study primarily focuses on the fluid flow in/out cartilage contact gap (Path 1, Fig.1), so that the simulations are initiated when highest asperities of two 
cartilage surfaces are about to (or just in) contact. The fluid permeability under different gap heights (simulation of gap closure) is estimated by applying pressure difference at the two ends of contact gap (Fig.2). To simplify the complicated fluid flow behaviour in the contact gap, the following assumptions are made in this study:

- The contact surface topography of two the cartilage surfaces are assumed to be symmetrical during contact. This assumption can greatly simplify the contact geometry without sacrificing the fundamental fluid flow behaviour in the contact gap.

- The fluid exchange between contact gap and cartilage tissue, and the bulk deformation of cartilage tissue are not considered in the study. This is because the gap permeability is related to the pore space in the contact gap, and the closing of the contact gap is primarily dependent on the asperity level deformation.

- Fluid-structure interaction in cartilage contact gap is not considered because it is not the primary focus of this study and its effect is probably minor. This is because solid matrices of asperities are incompressible, and in a pressure continuum space, the interstitial fluid pressure inside the asperity tissues tends to cancel out some of the outside fluid pressure in the contact gap. Therefore, the asperity deformation caused by fluid pressure in the gap is minimal compared to the external loading.

- The fluid flow in macro and micro scales can be modelled by Darcy's law and Naiver-Stokes equation, respectively. 
At the macro-scale, the contact gap between asperities of two cartilages forms interconnected pore spaces, and the gap flow behaviour can be described by Darcy's law in cylindrical coordinates,

$$
\boldsymbol{v}_{d}=-\frac{\kappa}{\mu} \nabla p=-K_{r} \frac{\Delta p}{\Delta r}
$$

where $K_{r}$ is the gap permeability, which represents the degree of difficulty for the flow to exit laterally. $\boldsymbol{v}_{\mathrm{d}}$ is the Darcy velocity of the gap flow. $p$ is the pressure of the fluid in the gap generated by the external load. $\mu$ is the viscosity of the synovial fluid. $k$ is the intrinsic permeability of the gap which is primarily dependent on the geometry of the pores in the gap, and in turn is function of the distribution of asperity size and gap height $h$ (i.e., $\kappa=\kappa(h)$ ). Since the centre line (mean) of the cartilage rough surface is usually taken at $z=0$, the gap height $h$ is defined as the distance between the centre lines of the upper and lower cartilage, which is also twice of the distance between the symmetrical line and the centre line of cartilage roughness in this study (Fig.2). Therefore, the gap height $h$ covers the positive asperity heights (peaks) of the upper and lower cartilage rough surface.

To guarantee statistical randomness, the selection of representative elementary volume (REV) for the micro-scale model is vital. In porous media, the REV is determined by gradually enlarging the averaging sample size to achieve relatively constant readings for variables (Bear, 1988). It is discovered that over a length scale of $250 \mu \mathrm{m}$ the asperities are relatively high and the peak heights are measured in around $10 \mu \mathrm{m}$. The maximum peak heights measured from square bovine knee joint samples of $250 \mu \mathrm{m}$ in length ranged from 6-18 $\mu \mathrm{m}$ (Graindorge et al., 2006). The levelled equine joint samples taken from three joint types (radiocarpal, midcarpal and carpometacarpal) with a length scale of $5000 \mu \mathrm{m}$ scanned by Smyth et 
al. (2012) showed that the asperity heights were around 5-10 $\mu \mathrm{m}$. This finding is consistent with the experimental measurements carried out in this study $\left(R_{\mathrm{p}}\right.$ value in Table 1 is close to $10 \mu \mathrm{m})$, which is based on a $1000 \mu \mathrm{m} \times 1000 \mu \mathrm{m}$ sample size. These findings imply that, at a contact length over $250 \mu \mathrm{m}$, the maximum gap size between two contact cartilages is around $20 \mu \mathrm{m}$. Therefore, in the present study a REV of 1000 $\mu \mathrm{m} \times 1000 \mu \mathrm{m}$ is considered as the suitable size for the cartilage contact problem. On the other hand, if we assume two primary roughly circular contact regions (lateral and media, each with a radius of $12-15 \mathrm{~mm}$, see Fig.1) are generated during standing or walking, the size of each contact area in the knee is around $500-700 \mathrm{~mm}^{2}$ (based on the contact radius of $12-15 \mathrm{~mm}$ ), compared to which the area of the REV is $1 / 700-$ 1/500. This implies the chosen REV size is appropriate to the scale for the in vivo knee cartilage contact problem.

Isothermal, laminar, incompressible flow conditions are assumed in the gap for the 203 micro-scale REV model ( $1 \mathrm{~mm} \times 1 \mathrm{~mm}$ square). The flow is governed by the Navier204 Stokes equations in Cartesian coordinates.

205

$$
\left\{\begin{array}{l}
\nabla \cdot \boldsymbol{u}=0 \\
\rho \frac{\partial \boldsymbol{u}}{\partial t}+\rho(\boldsymbol{u} \cdot \nabla) \boldsymbol{u}=\nabla \cdot[-p \boldsymbol{I}+\mu(\dot{\gamma}) \cdot \dot{\gamma}]+\boldsymbol{F}
\end{array}\right.
$$

where $\rho$ is the density of synovial fluid; $\boldsymbol{u}$ is fluid velocity vector; $p$ is fluid pressure;

$\boldsymbol{F}$ is the volume force vector which is ignored in this study; $\mu$ is the viscosity of synovial fluid. $\dot{y}$ is the shear rate tensor, its expression and its magnitude shear 209 rate $\dot{y}$ are given below,

$$
\begin{aligned}
& \dot{\gamma}=\nabla \boldsymbol{u}+(\nabla \boldsymbol{u})^{T} ; \\
& \dot{\gamma}=|\dot{\gamma}|=\sqrt{\frac{1}{2} \dot{\gamma}: \dot{\gamma}}
\end{aligned}
$$


211 Recent studies of the synovial fluid flow in the contact gap mainly treated synovial fluid as a Newtonian fluid with constant viscosity (e.g. 1 Pa.s was used by Wu and Ferguson, 2017, and 0.6 Pa.s by Moghadam et al., 2014). However, synovial fluid is a non-Newtonian fluid with a shear-thinning effect, i.e., the viscosity decreases with increasing shear rate (Fam et al., 2007). The relationship between viscosity and shear rate is usually obtained from viscometer experiments on oscillating flow, in which the synovial fluid is pre-sheared for two minutes, and then different shear rates are applied to the sample until the steady state is reached (Bhuanantanondh, 2009). Initially, the viscosity of synovial fluid is high due to the randomly orientated long molecules, then it decreases as the long molecules are re-orientated and stretched in the direction of the flow lines. In this study, the viscosity of synovial fluid is defined by gap height and fluid velocity (i.e., $\mu=\mu(h, u)$ ) through relating the shear rate to the gap height and fluid velocity as per Eq. (3). In addition, synovial fluid also exhibits time dependent shear thickening. To remove timea shear thinning fluid.

In order to better capture the gap flow behaviour, two major inputs are incorporated in the micro-scale REV model.

1. Experimentally measured and numerically synthesised cartilage surface roughness.

2. Constitutive relationship for synovial fluid by curve-fitting the experimental data of steady state oscillating flow. 
234 The link from the micro to macro scale is drawn by equating the volume235 averaged velocity of the REV (microscale) to the Darcy velocity in macro scale. 236 Thus, the macro scale gap permeability $K_{r}$ can be evaluated.

237

$$
u_{\text {vol.ave }}=\frac{1}{V} \int_{R E V} u_{i} \cdot d V_{i}=v_{d}
$$

$$
K_{r}=v_{d} \frac{\Delta r}{\Delta p}=u_{\text {vol.ave }} \frac{\Delta r}{\Delta p}
$$


Contact moving downwards

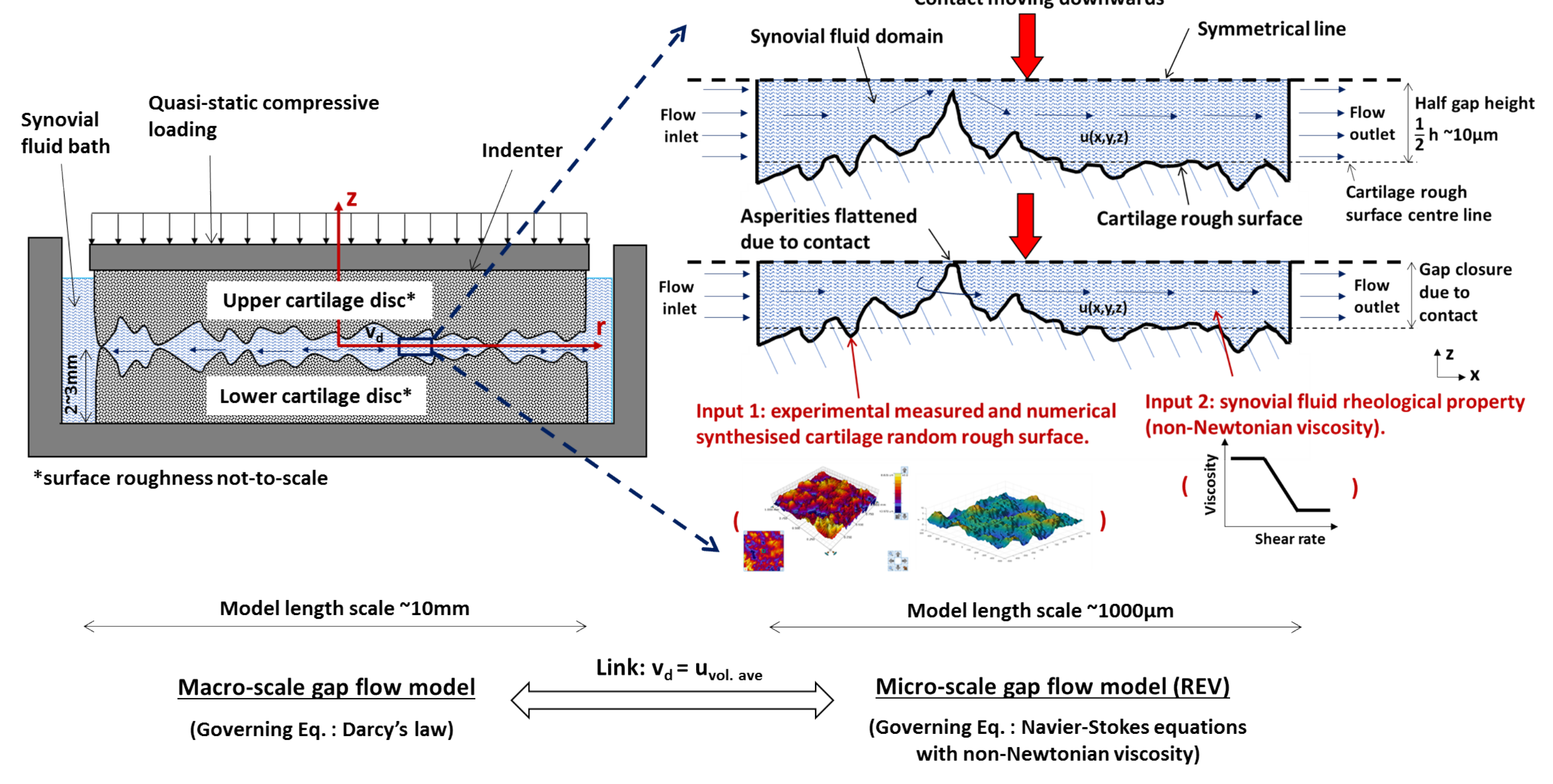




\subsection{Reconstruction of cartilage surface topography}

\subsubsection{Experimental measurements of cartilage surface roughness}

Bovine patellofemoral joints $(n=3)$ of 2 to 4 -year-old animals were sourced from abattoir and were dissected to obtain fresh tibias with microscopically normal cartilage. The femurs were soaked in $0.15 \mathrm{M}$ Phosphate-buffered saline solution and stored at minus 20 degrees $\mathrm{C}$.

Prior to harvesting the osteochondral sample blocks (approximately $1 \mathrm{~cm} \times 1 \mathrm{~cm}$ ) form lateral femur, the intact femur was thawed in $0.15 \mathrm{M}$ Phosphate-buffered saline at room temperature. The osteochondral blocks were then glued (LOCTITE $®$ SUPER GLUE GEL CONTROL ${ }^{\mathrm{TM}}$ ) on a glass slide (Fig.3 (b)) and were loaded on to the stage of a stylus profiler (DektakXT, Bruker) (Fig.3 (a)) for the roughness measurements.

Surface scanning was conducted using a $2 \mu \mathrm{m}$ tip over an area of $1 \mathrm{~mm} \times 1 \mathrm{~mm}$, approximately at the middle of the sample. Styles force of $1 \mathrm{mg}$ was used for the scanning and samples were kept hydrated throughout. The scanning speed was set to $100 \mu \mathrm{m} / \mathrm{s}$ with a Y-resolution of $0.33 \mu \mathrm{m} / \mathrm{pt}, 200$ scans in the $X$-direction and vertical resolution (Z-resolution) of 8nm (Fig.3 (c)). Each sample took approximately 40 mins to scan and the 3D scanned profiles were analysed using DektakXT inbuilt Vision64 software to obtain the roughness parameters of the cartilage

The cartilage surface topographies medial tibia are shown in Fig.4 and the surface parameters are summarised in Table 1. Previous studies on cartilage surface roughness have shown that roughness height is dependent on the size of the cartilage specimen (i.e., length scale), with increasing size of a specimen, the values of $R_{\mathrm{a}}$ and $R_{\mathrm{q}}$ generally increase. For small length scales which are less than $50 \mu \mathrm{m}, R_{\mathrm{q}}$ is measured in hundreds of nanometres. For example, Lee et al. (2012) measured 
264 human femoral heads $(40 \mu \mathrm{m} \times 40 \mu \mathrm{m})$ with different osteoarthritis stages and it is 265 showed that the values of $R_{\mathrm{q}}$ were in a range of $137 \mathrm{~nm}$ to $533 \mathrm{~nm}$. In addition, the 266 study of Graindorge et al. (2006) on $250 \mu \mathrm{m} \times 250 \mu \mathrm{m}$ square samples harvested from 267 healthy mature bovine knee joint showed that the values of $R_{\mathrm{a}}$ and $R_{\mathrm{q}}$ were $1.06 \mu \mathrm{m}$ 268 and $1.32 \mu \mathrm{m}$, respectively. Furthermore, the measurements of the cartilage surface $269(702 \mu \mathrm{m} \times 527 \mu \mathrm{m})$ of 6 to 8 -month-old bovine knee joints by Shekhawat et al. (2009) 270 showed that the trochlea $R_{\mathrm{a}}$ was $1.15 \mu \mathrm{m}$ and chondyle $R_{\mathrm{a}}$ was $0.6 \mu \mathrm{m}$. Later on,

271 Smyth et al. (2012) measured three types of equine joints with a length scale of 5000 $272 \mu \mathrm{m}$. It is showed that the values of $R_{\mathrm{a}}$ for midcarpal, carpometacarpal and radiocarpal 273 joints were $2.29 \mu \mathrm{m}, 1.69 \mu \mathrm{m}$ and $1.60 \mu \mathrm{m}$, respectively. Therefore, by comparison, 274 the surface roughness measurements obtained in this study $\left(R_{a}=1.97 \mu m, R_{q}=2.44\right.$ $275 \mu \mathrm{m}$ for a specimen size of $1000 \mu \mathrm{m} \times 1000 \mu \mathrm{m})$ are in reasonable agreement with 276 previous measurements. 


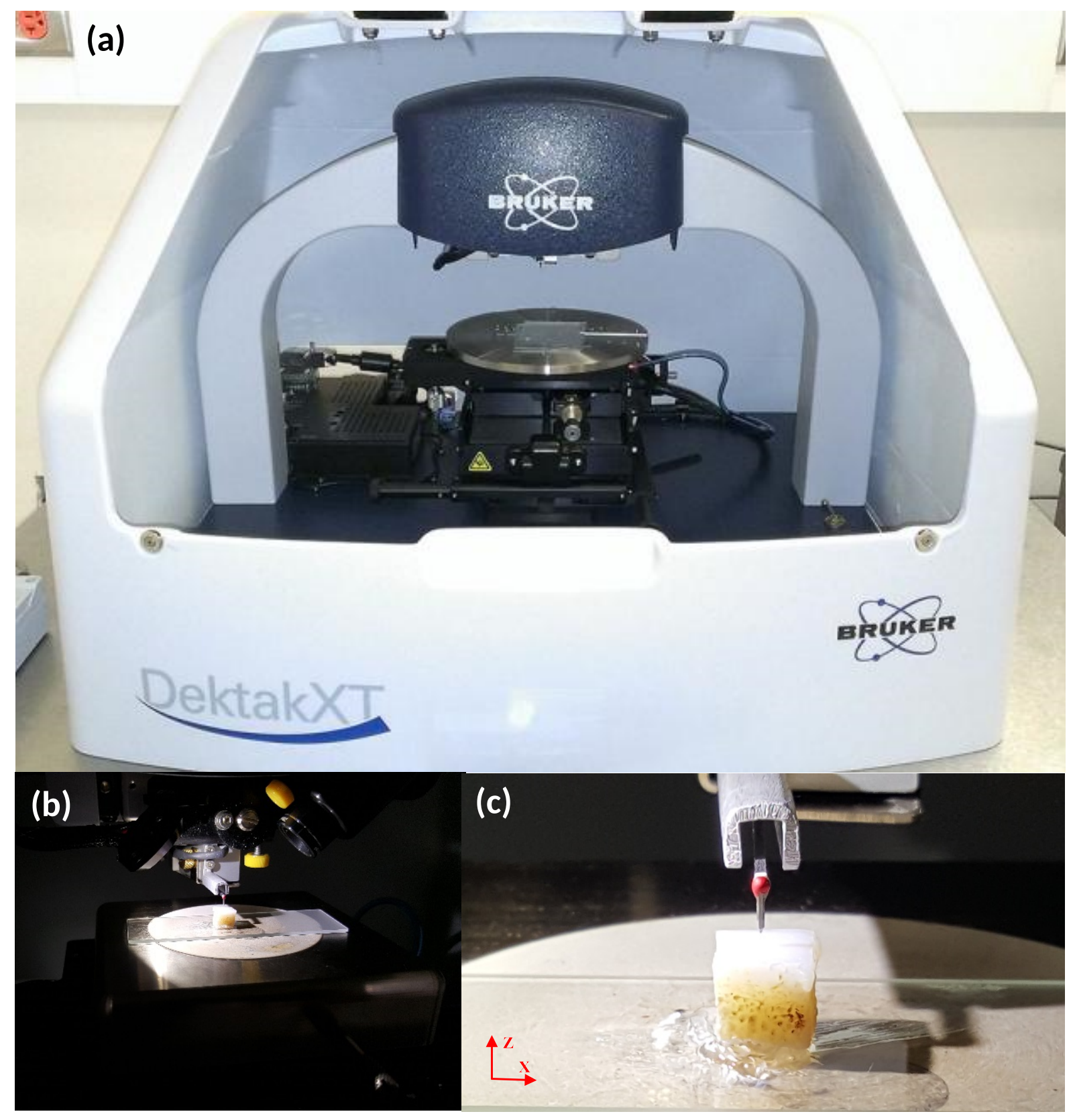

Fig.3 - Experimental equipment and samples: (a) DektakXT, Bruker Styles profiler; (b) Glued osteochondral blocks loaded on the styles profiler; (c) The scanning directions and close-up view of the hydrated, glued osteochondral block and the styles tip location. 


\subsubsection{Synthesis of cartilage surface with random roughness}

Although experimental measurements can provide exact surface roughness, the costs, time and workloads are tremendous especially for multiple sample statistical analyses. On the other hand, the characterisation of rough surfaces can also be, more conveniently, synthesised by numerical methods, by which means multiple random samples can be generated swiftly with desired surface parameters. In this study, a numerical procedure has been utilised to synthesise rough cartilage surfaces that resemble the experimental measurements in key parameters (i.e., $R_{\mathrm{q}}$ and correlation length).

Traditionally, the surface roughness is quantified by RMS roughness height, which only describes the vertical roughness (i.e., asperity heights) (Smyth et al., 2012). In order to capture the whole picture of cartilage surface roughness, another parameter "correlation length" is required to describe the horizontal roughness (i.e., wavelength) constructing the semi-variogram for the surface. The mathematical definition of semivariogram $y$ is given below,

$$
\gamma(\Delta x, \Delta y)=\frac{1}{2} E\left\{[z(x+\Delta x, y+\Delta y)-z(x, y)]^{2}\right\}
$$
where $z(x, y)$ is the surface heights; $E\{\}$ is the statistical expectation operator; $\Delta x$ and $\Delta y$ are the lag distance in two orthogonal directions. Based on the definition, if the lag distance is chosen to be very small, the two points are located very closely on the same bump, and hence the variogram $\gamma$ value computed by Eq. (5) is small, and it tends to increase with the lag distance until it reaches a bend down (i.e., transition) area that the dependence of variogram on lag distance becomes diminished, which 
indicates the two points gradually lose correlation. After that the two points are no longer related and the variogram exhibits a plateau called the sill. Theoretically the lag distance corresponding to this bend down area can be taken as the correlation length. In this study, the semi-variograms are computed by the 3D mapping software Surfer (version 15, Golder Software LLC), and the semi-variogram is fitted to an exponential function, so the correlation length corresponds to the distance at which the variance is $63 \%$ of the sill (Barnes, 2003).

As observed from the experimental results, the sample surface is primarily composed of "secondary undulations" and "tertiary undulations" as per the classification described by Longmore and Gardner (1975). "Secondary undulations" are asperities with a length scale of $200-500 \mu \mathrm{m}$ and an average height around 10-15 $\mu \mathrm{m}$, while "tertiary undulations" are the next size lower that length scale varies from 20 to $40 \mu \mathrm{m}$, and asperity heights are $0.5-2 \mu \mathrm{m}$. In order to better simulate the cartilage surface, the information of the secondary and tertiary length scales shall be captured. This is achieved by the following steps:

1) Decompose the measured surface into secondary and tertiary undulations by applying Gaussian 2nd order regression filter (see Fig.4 (a)).

2) Obtain the key surface parameters (i.e., $R_{\mathrm{q}}$ and correlation length) from both secondary and tertiary undulations (see Fig.4 (a)).

3) Generate two sperate random rough surfaces with the obtained key surface parameters to reflect the secondary and tertiary undulations respectively by using the numerical procedures given below.

4) Superpose the synthetic secondary and tertiary undulation surfaces to form a completed cartilage random rough surface (see Fig.4 (b)). 

method combined with the power spectra density function (Wu, 2000). According to Bergström et al. $(2007,2008)$, a three dimensional random rough surface, described by $z=z(x, y)$, can be generated using two statistical distribution functions: the height probability distribution function (HDF) $P(z)$ and the auto-correlation function (ACF) $R_{\mathrm{zz}}(x, y)$. The height probability distribution function characterises how far the probability of the surface heights deviate from the mean value (usually taken as $z=0$ ). The auto-correlation function describes how strongly a certain surface height is correlated to another surface height separated by the 'lag distance', by defining the correlated length. In short, the HDF characterises the amplitudes of the surface heights, while the ACF describes the wavelengths of the peaks and valleys of the rough surfaces. It is common practice to assume both HDF and ACF follow the Gaussian distribution as follows,

where $\sigma$ is the standard deviation, which is the RMS roughness height in this study (i.e., $\sigma=R_{\mathrm{q}}$ ). $\tau_{\mathrm{x}}$ and $\tau_{\mathrm{y}}$ are the correlation lengths in $\mathrm{x}$ and $\mathrm{y}$ directions respectively. As stated above, the correlation lengths are computed by constructing the semivariograms shown in Fig.4 (a), in which isotropic correlation lengths of $100 \mu \mathrm{m}$ and $20 \mu \mathrm{m}$ are obtained from the secondary and tertiary undulations respectively. to generate the random surface roughness. The procedures are briefly described below. 
On discrete mesh points of the $x-y$ plane, a series of uncorrelated but normally

357

358

359

360

361

362

363

364

365

366

367

368

369

370

371

372

373

374

distributed random surface heights $\left\{\eta_{x, y}\right\}(\mathrm{N} \times \mathrm{M}$, in which $\mathrm{N}$ and $\mathrm{M}$ are the number of surfaces points in $\mathrm{x}$ and $\mathrm{y}$ directions respectively) are generated by Eq. (6) with a given mean value $(z=0)$ and standard deviation $\sigma$.

Performing the 2D FFT of $\eta_{x, y}$ yields the frequency contents (or the wavelength information) of the series $\eta_{\mathrm{x}, \mathrm{y}}$ denoted by $A\left(\omega_{\mathrm{x}}, \omega_{\mathrm{y}}\right)$.

$$
A\left(\omega_{x}, \omega_{y}\right)=\sum_{n=0}^{N-1} \sum_{m=0}^{M-1} \eta_{x, y} \cdot e^{-i k \omega_{x}} \cdot e^{-i k \omega_{y}}, \quad\left(\omega_{x}=\frac{2 \pi n}{N}, \omega_{y}=\frac{2 \pi m}{M}\right)
$$

The power spectral density function of $\eta_{x, y}$ is given by Eq. (9),

$$
S_{\eta \eta}\left(\omega_{x}, \omega_{y}\right)=\left|A\left(\omega_{x}, \omega_{y}\right)\right|^{2}
$$

where $k$ and $/$ are variable integers from 0 to $N-1$ and $M-1$ respectively.

Computing the 2D FFT of the ACF $R_{\mathrm{zz}}(x, y)$ gives the power spectra density function of the rough surface heights $S_{z z}\left(\omega_{x}, \omega_{y}\right)$ in Eq. (10)

$$
S_{z z}\left(\omega_{x}, \omega_{y}\right)=\sum_{n=0}^{N-1} \sum_{m=0}^{M-1} R_{z z}(x, y) \cdot e^{-i k \omega_{x}} \cdot e^{-i k \omega_{y}}
$$

The frequency contents (or the wavelength information) of the correlated rough surface $Z\left(\omega_{x}, \omega_{y}\right)$ can be obtained by computing the complex products of two series $A\left(\omega_{\mathrm{x}}, \omega_{\mathrm{y}}\right)$ and $H\left(\omega_{\mathrm{x}}, \omega_{\mathrm{y}}\right)$, where $H\left(\omega_{\mathrm{x}}, \omega_{\mathrm{y}}\right)$ is the transfer function of the system that is shown in Eq. (11).

$$
\begin{aligned}
& H\left(\omega_{x}, \omega_{y}\right)=\sqrt{\frac{S_{z z}\left(\omega_{x}, \omega_{y}\right)}{S_{\eta \eta}\left(\omega_{x}, \omega_{y}\right)}} \\
& Z\left(\omega_{x}, \omega_{y}\right)=H\left(\omega_{x}, \omega_{y}\right) \cdot A\left(\omega_{x}, \omega_{y}\right)
\end{aligned}
$$

Finally, the correlated normally distributed rough surface heights can be computed by an inverse fast fourier transform (IFFT) of $Z\left(\omega_{x}, \omega_{y}\right)$. 


$$
z(x, y)=\frac{1}{N M} \sum_{n=0}^{N-1} \sum_{m=0}^{M-1} Z\left(\omega_{x}, \omega_{y}\right) \cdot e^{i k \omega_{x}} \cdot e^{i k \omega_{y}}
$$

The algorithm has been implemented in MATLAB (R2017b, The MathWorks, Inc.). 10 samples were generated for cartilage on medial tibia. The comparison of surface parameters is summarised in Table 1. Additionally, the synthetic rough surfaces with the closest $R_{\mathrm{q}}$ values out of the 10 samples to the corresponding experimental measurements is plotted in Fig.4 (b).

Generally, the key parameters of the synthesised surfaces match well with the experimental measurements, especially for the averaged roughness $R_{\mathrm{a}}$ and RMS roughness $R_{\mathrm{q}}$ values. The maximum differences are less than $1 \%$. It should be noted that the mathematical algorithm is a pseudo-random process and hence the synthetic rough surfaces exhibit more evenly distributed values for surface peaks and valleys than the measured samples. Therefore, there are small discrepancies for the comparison of the maximum peak height $R_{\mathrm{p}}$ and maximum valley depth $R_{\mathrm{v}}$. This is believed to be caused by the sample sizes. With larger sample sizes, the key parameters can be captured more accurately. However, 10 samples are considered sufficient in this study to satisfy statistical requirements based on the standard deviation convergence tests for viscosity and permeability in Section 3.2.

Table 1 - Surface roughness parameter comparisons between experiment and synthetisation

\begin{tabular}{ccc}
\hline Surface & \multicolumn{2}{c}{ Medial Tibia } \\
\cline { 2 - 3 } parameter & Experiment & Synthetisation* \\
\hline$R_{\mathrm{a}}(\mu \mathrm{m})$ & 1.97 & $1.94(0.069)$ \\
$R_{\mathrm{q}}(\mu \mathrm{m})$ & 2.44 & $2.42(0.074)$ \\
$R_{\mathrm{p}}(\mu \mathrm{m})$ & 8.83 & $7.66(0.313)$ \\
$R_{\mathrm{v}}(\mu \mathrm{m})$ & -13.92 & $-7.29(0.756)$ \\
$R_{\mathrm{t}}(\mu \mathrm{m})$ & 22.75 & $14.95(0.723)$ \\
\hline
\end{tabular}

*values outside the brackets are means, inside the brackets are standard derivations $(n=10)$. 

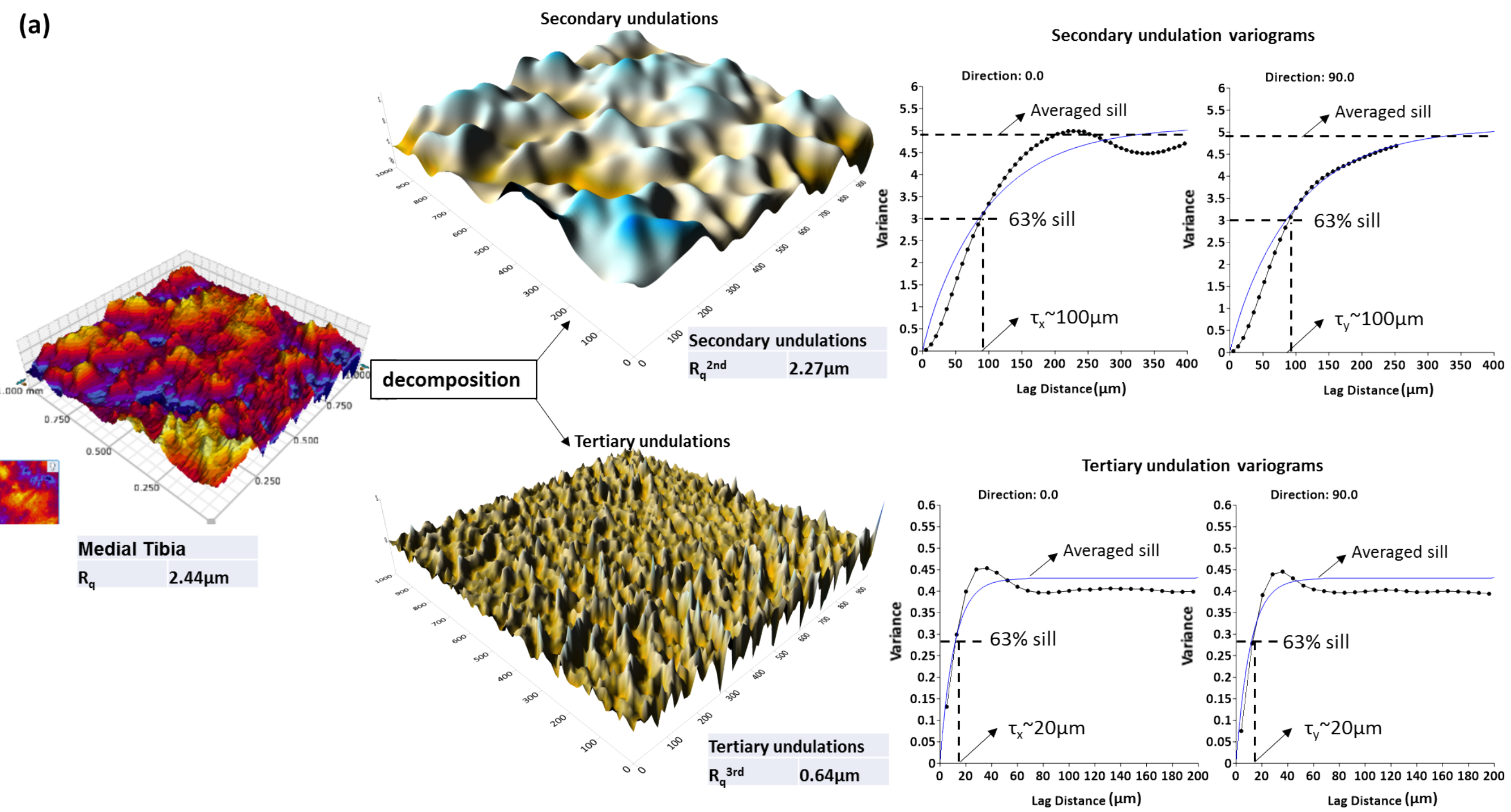
(b)
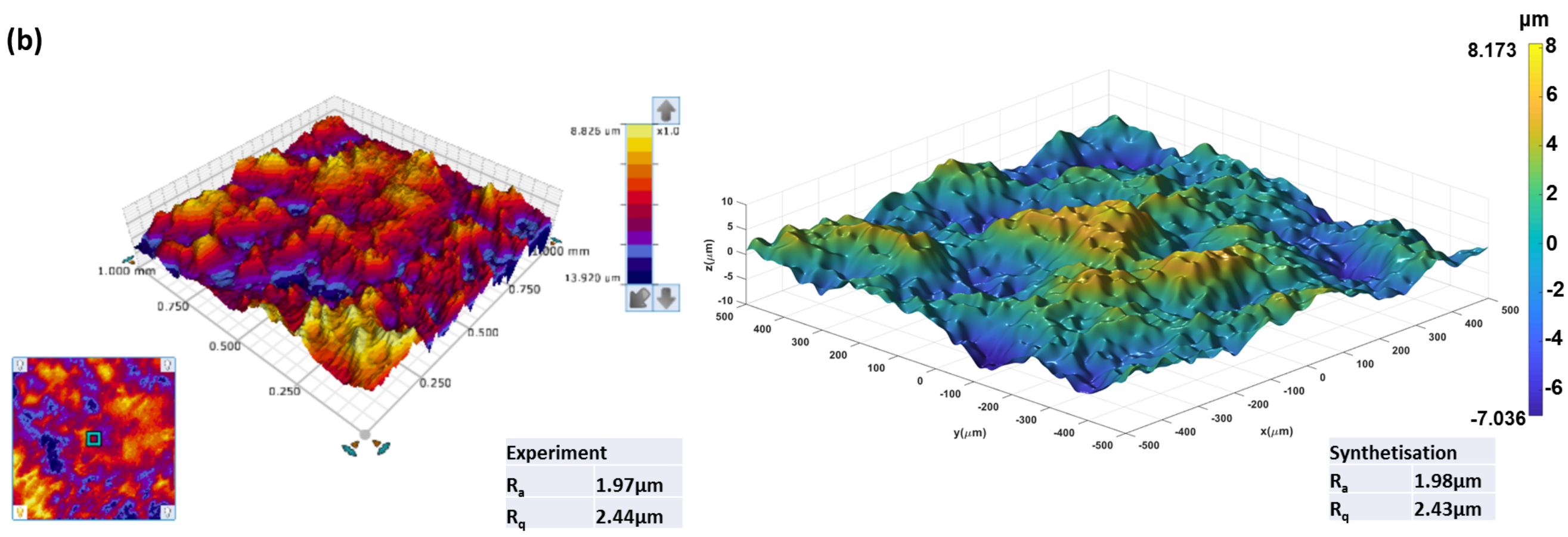

$\mathrm{R}_{\mathrm{a}} \quad 1.98 \mu \mathrm{m}$

$\begin{array}{ll}\mathrm{R}_{\mathrm{a}} & \mathbf{2 . 4 4 \mu \mathrm { m }}\end{array}$

$\begin{array}{ll}R_{\mathrm{q}} & 2.43 \mu \mathrm{m}\end{array}$

Fig.4 - Synthesise cartilage rough surfaces based on experimental measurements: (a) Decomposed secondary \& tertiary undulations and variograms in two orthogonal directions; (b) Cartilage topography and synthesised random rough surface. 


\subsection{Numerical analysis of contact gap flow}

\subsubsection{Model description}

The simulations of fluid flow within the gap between rough opposing surfaces were performed in COMSOL Multiphysics (version 5.3, COMSOL, Inc.). A typical microscale REV is shown in Fig.5 (a). The random rough surfaces (lower wall) generated above were imported to COMSOL.

The upstream and downstream walls (highlighted in blue) in the x direction were defined as inlet and outlet boundaries, respectively. The two lateral walls (highlighted in yellow) in the y direction were symmetrical boundaries parallel to the flow to form a continue pore space. The lower surface representing the cartilage roughness in z direction was assigned no-slip boundary. In addition, the upper wall in z direction was defined as symmetry boundary to simulate two symmetrical cartilage contact. Fluidstructure interactions between the gap flow and the asperities were not considered, as it is probably a minor effect and not the primary focus of this study.

However, the analyses did consider the consolidation of the asperities in the pore spaces. Consolidation of the asperities alters the size and shape of the gap. Since the Poisson's ratio of the cartilage extracellular matrix often approaches zero (Mow et al., 1993), especially in deformation rate regimes in which the interstitial fluid within the asperity has time to leave the tissue (there is fast consolidation as a result of the short asperity drainage path length)). It is convenient to approximate the gap closure geometry by just lowering down the contact plane to intersect with the asperities at varies heights (i.e., at 8, 6, 4, 2 and $0.5 \mu \mathrm{m}$ for a single cartilage, which corresponds to the total gap heights of $16,12,8,4$ and $1 \mu \mathrm{m}$ respectively for the contact of two cartilages with the same topography). The cartilage surface roughness at varies heights and degrees of contact are shown in Fig.5 (c). Separate models were created 
430 for each gap height, and for each gap model, various inlet pressures, ranging from 431100 to10000 $\mathrm{Pa}$, together with the outlet pressure set at zero Pascal were applied. Laminar, incompressible and isothermal flow was assumed in the gap for the microscale REV models. Stationary Navier-Stokes equations were solved by the generalised minimal residual method (GMERS) for each gap model at each inlet pressure.

\subsubsection{Viscosity of synovial fluid}

Synovial fluid is a semidilute hyaluronate (HA) and protein solution with a density of $1225 \mathrm{~kg} / \mathrm{m}^{3}$, the viscosity of which exhibits non-Newtonian shear rate dependent characteristics. The viscosity of synovial fluid will affect the flow velocity in the gap. The rheology of synovial fluid has been studied extensively (Fam et al., 2007). The experimental results by Schurz and Ribitsch (1987) showed the zero-shear rate viscosity $\left(\mu_{0}\right)$ ranges for healthy synovial fluid were 1-40 Pa.s. After a Newtonian plateau at shear rate around $0.05-0.1 \mathrm{~s}^{-1}$, synovial fluid exhibited shear thinning behaviour up to shear rate of $1000 \mathrm{~s}^{-1}$ where the reported experimental data terminated, at which the corresponding viscosity for healthy synovial fluid is 0.009 446 Pa.s.

The generalised Newtonian constitutive equations have been used to capture the non-Newtonian behaviour of synovial fluid. The curve-fitting power law equation reported by Lai et al. (1978) indicated that a normal synovial fluid would reach the infinite shear viscosity $\left(\mu_{\infty}\right)$ around $0.007-0.01 \mathrm{~Pa} \cdot \mathrm{s}$ for high shear rates more than $4511000 \mathrm{~s}^{-1}$.

The present study combined the findings of these two studies (Schurz and 
454 experimental data of the healthy synovial fluid by Schurz and Ribitsch (1987), in 455 which the shear thinning was approximated by a power law equation between the 456 two Newtonian plateaus of zero-shear viscosity $\mu_{0}=20.3 \mathrm{~Pa} \cdot \mathrm{s}$ and infinite-shear 457 viscosity $\mu_{\infty}=0.01 \mathrm{~Pa} \cdot \mathrm{s}$ as per Lai et al. (1978). The equation is present below and 458 plotted in Fig.5 (b) for comparison.

459

$$
\mu(P a \cdot s)=\left\{\begin{array}{ccc}
20.3 & , & \left(\dot{\gamma} \leq 0.05 s^{-1}\right) \\
m \dot{\gamma}^{n-1}(m=2.237, n=0.263) & , & \left(0.05 s^{-1} \leq \dot{\gamma} \leq 1000 s^{-1}\right) \\
0.01 & , & \left(\dot{\gamma} \geq 1000 s^{-1}\right)
\end{array}\right.
$$

460 It should be noted that the experimental data measured by viscometers is usually 461 from steady state oscillating flow, as the synovial fluid samples are usually pre462 sheared for a few minutes before testing. The oscillating shear test is not exactly 463 the same as the conditions in a joint, but it is probably the closest representation 464 for a cyclically loaded joint. 
(a)

Symmetrical

boundary

(side wall)

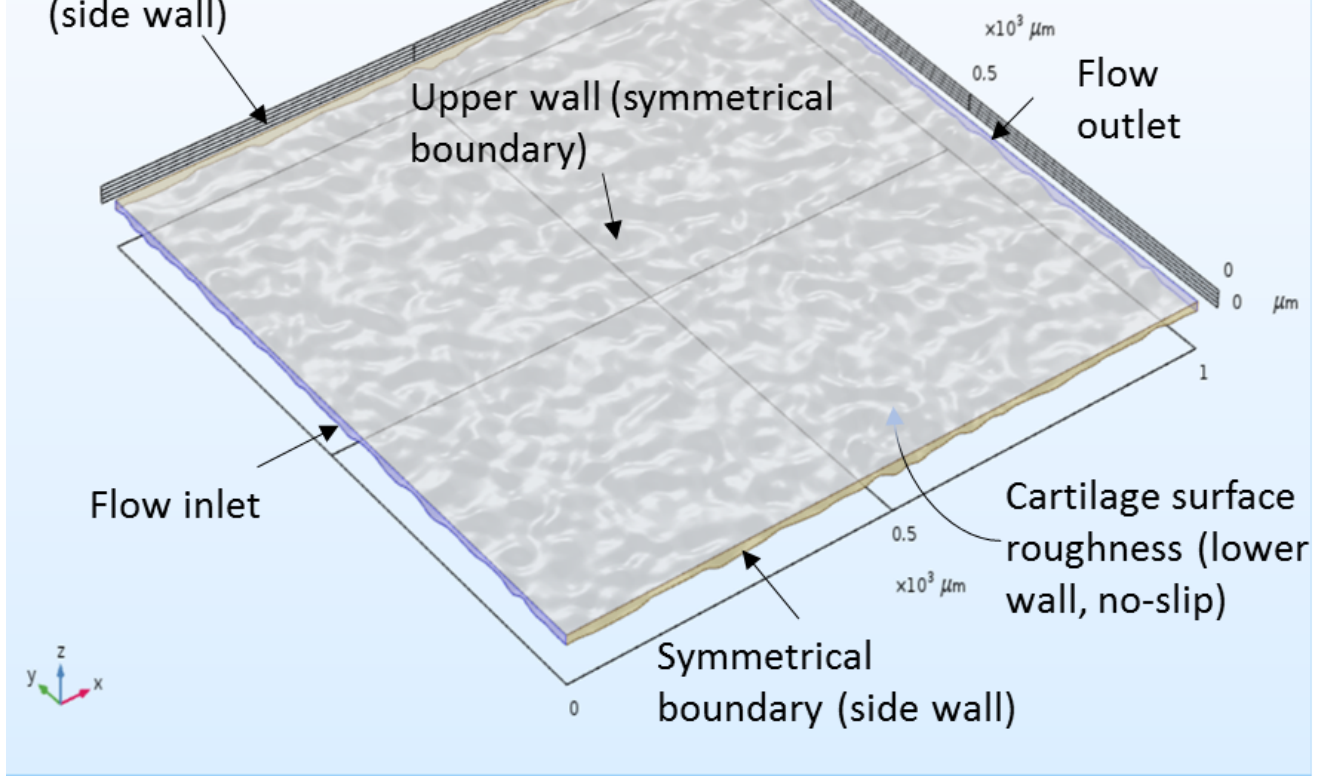

(b)

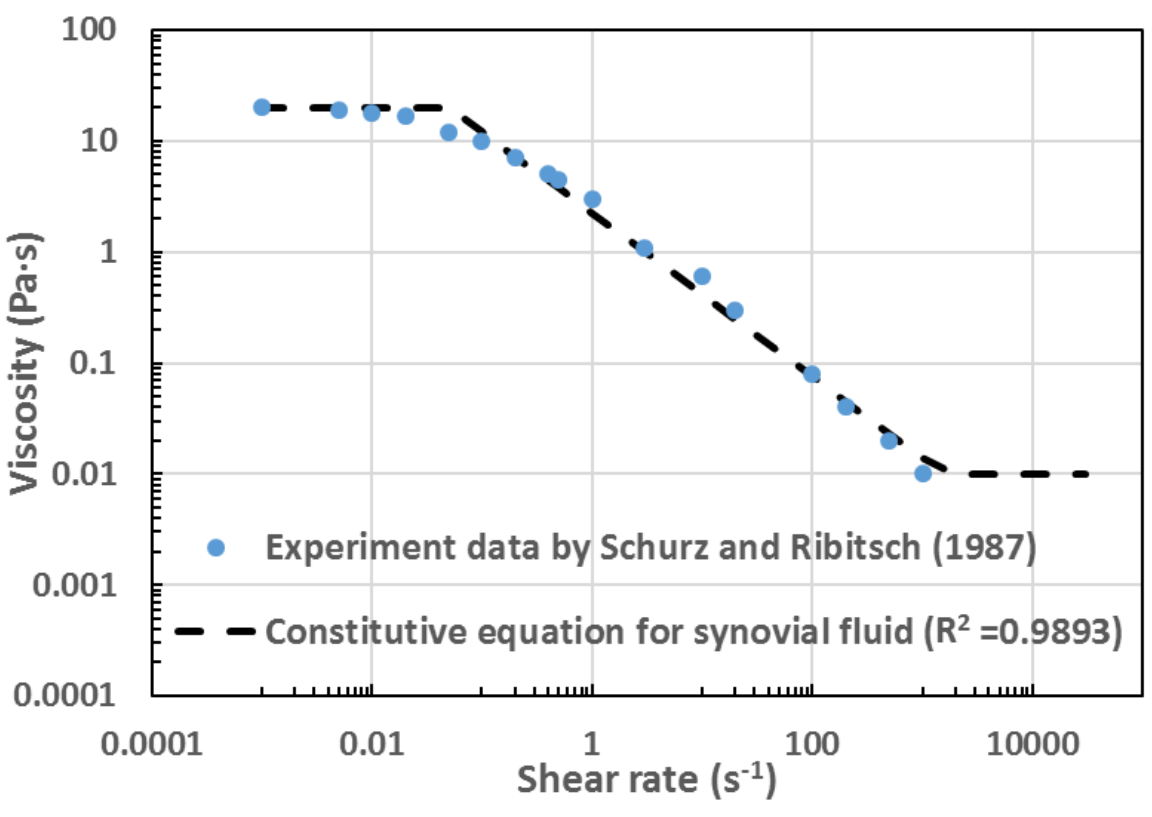


(c)

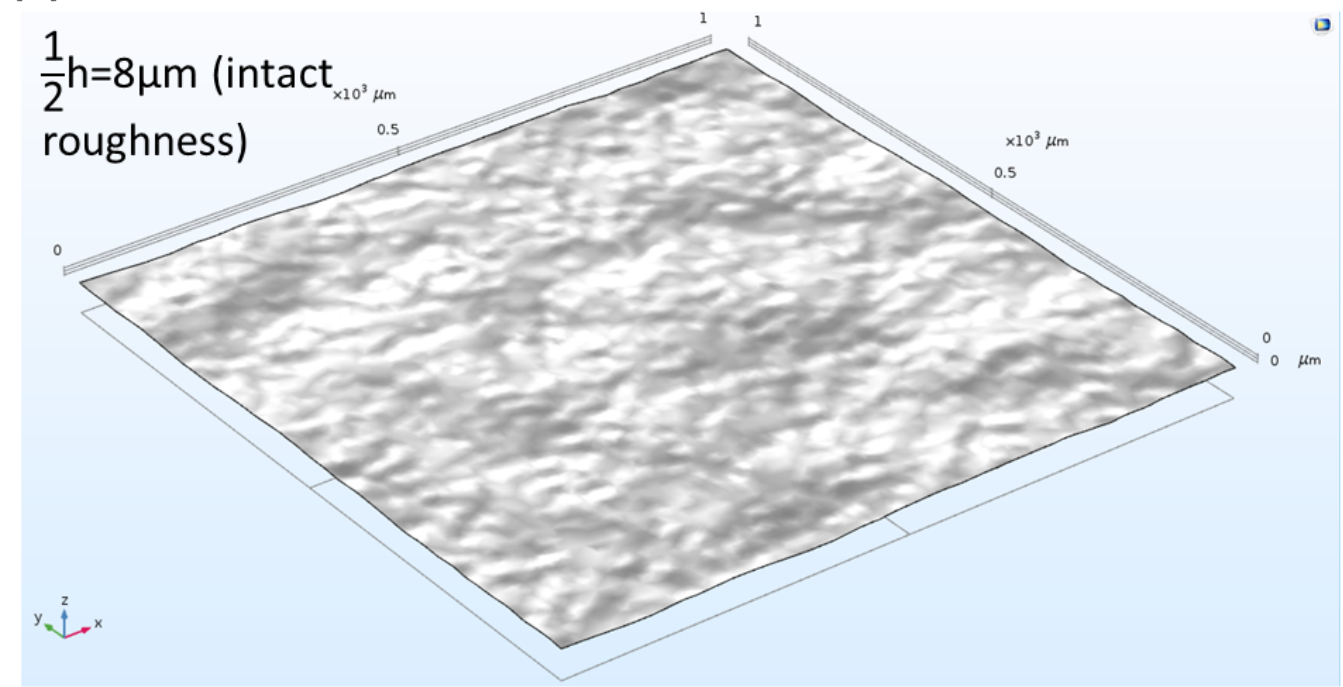

$\frac{1}{2} h=2 \mu m$

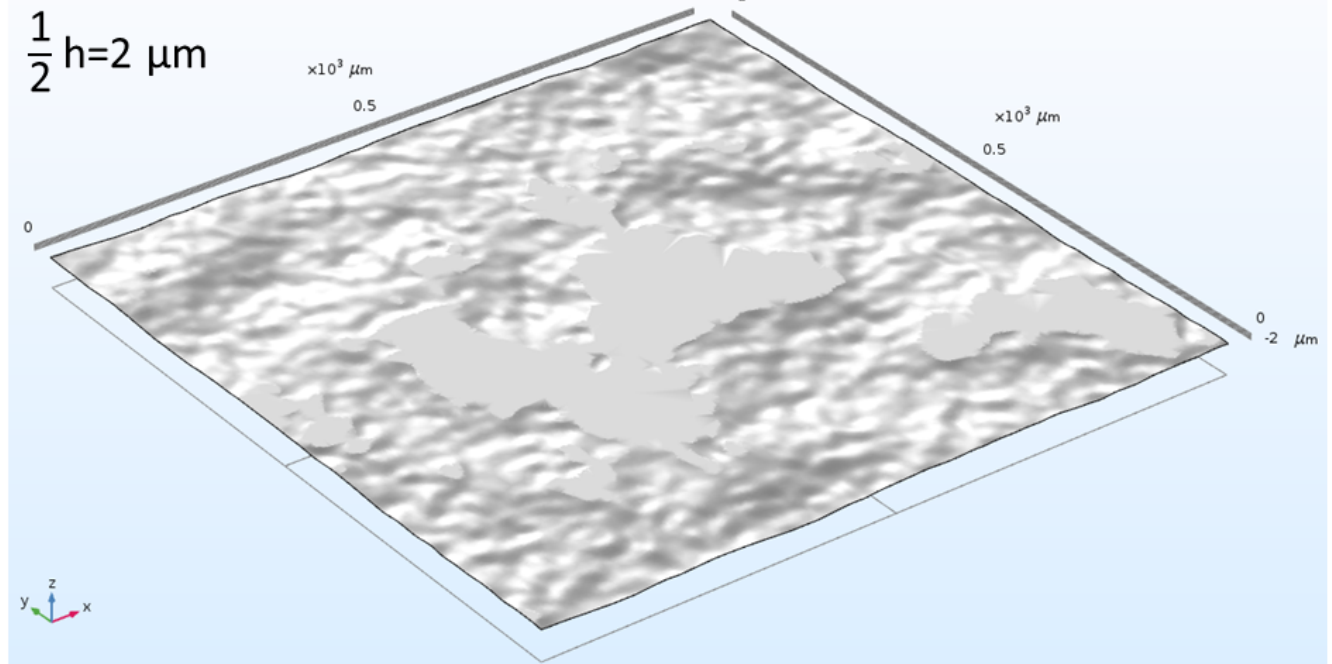

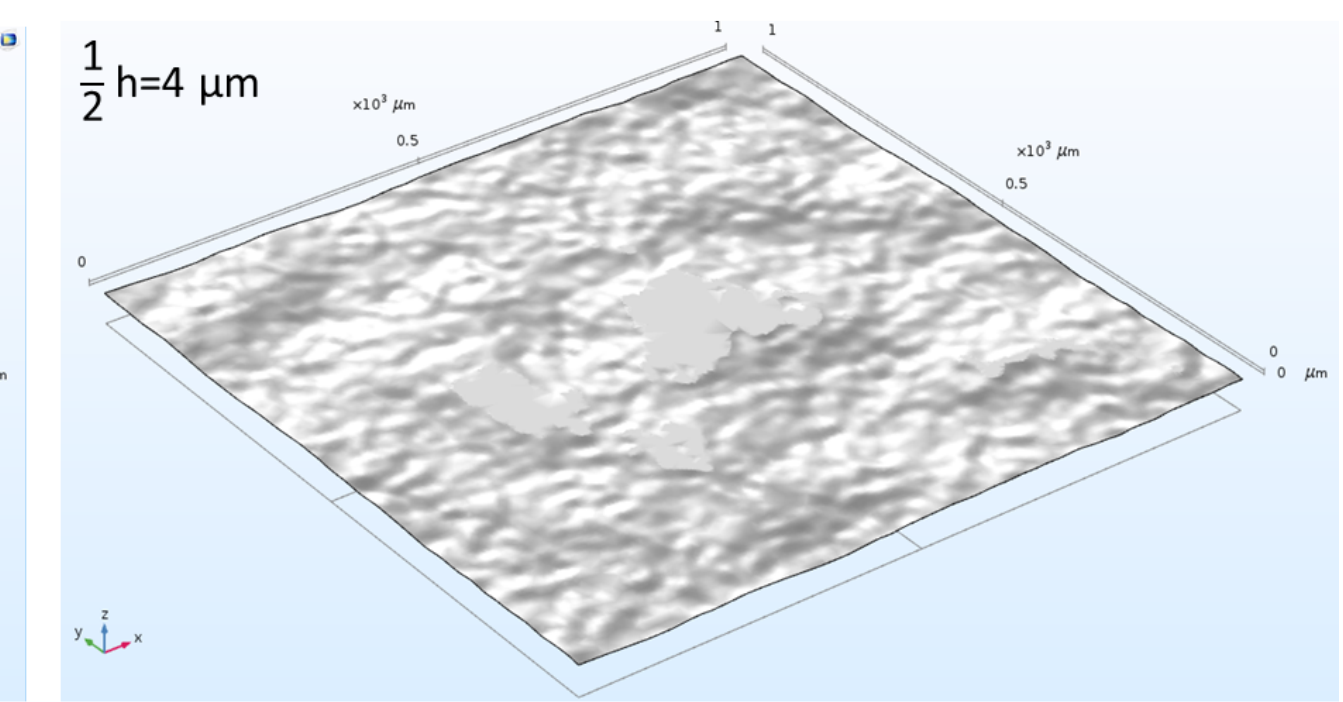

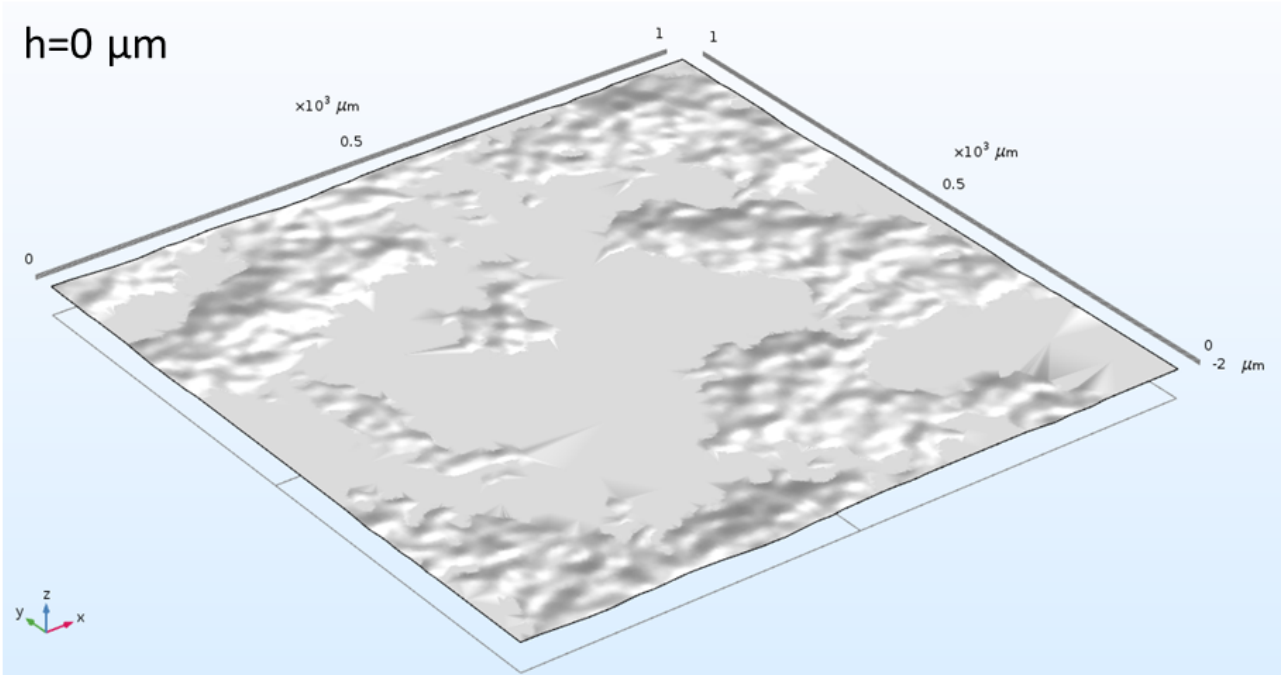

Fig.5 - Numerical analyses setup: (a) A typical micro-scale REV model for CFD analyses; (b) Comparison of synovial fluid constitutive equation with experimental data; (c) Shapes of the cartilage surface roughness at different gap heights. 


\section{RESULTS AND DISCUSSION}

471

472

473

474

475

476

477

478

479

480

481

482

483

484

485

486

487

488

489

490

491

\subsection{Initial tests}

Initial testing was performed for the purposes of result comparison and model verification against the previous findings.

The initial analysis inputs were set as close as possible to mimic Wu and Ferguson (2017) simulations, i.e., fluid domain was set as $80 \mu \mathrm{m} \times 80 \mu \mathrm{m}$, RMS roughness $R_{\mathrm{q}}$ $=0.5 \mu \mathrm{m}$, inlet pressure was $400 \mathrm{~Pa}$, outlet pressure was $0 \mathrm{~Pa}$ and fluid viscosity was $1 \mathrm{~Pa}$. . However, the wavelength information was not provided, to this end, three surfaces with different correlation lengths (i.e., $\mathrm{T}=2,4$ and $6 \mu \mathrm{m}$ ) were generated for comparison.

The volumetric averaged velocities were output at various gap heights. The gap permeability $K_{r}$ was calculated using Eq. (4) and results are plotted in Fig.6 for comparison. As viscosity is constant, $K_{r}$ is only related to the intrinsic permeability $K$ which is a function of pore size (i.e., gap height $h$ ). It is shown that generally the results of initial tests match closely with Wu and Ferguson (2017) both in trend and absolute value. Through the calibration process, it is shown that the results of $\mathrm{T}=6 \mu \mathrm{m}$ match the most closely to the results of Wu and Ferguson (2017) (less than 4\% difference).

Another noticeable trend is that surfaces with smaller correlation lengths tend to have better fluid retention capability. This is because under the same REV size and $R_{\mathrm{q}}$, smaller correlation lengths generate more undulations that enlengthens the drainage path. The results underline the importance of considering the two correlation lengths in the model. 


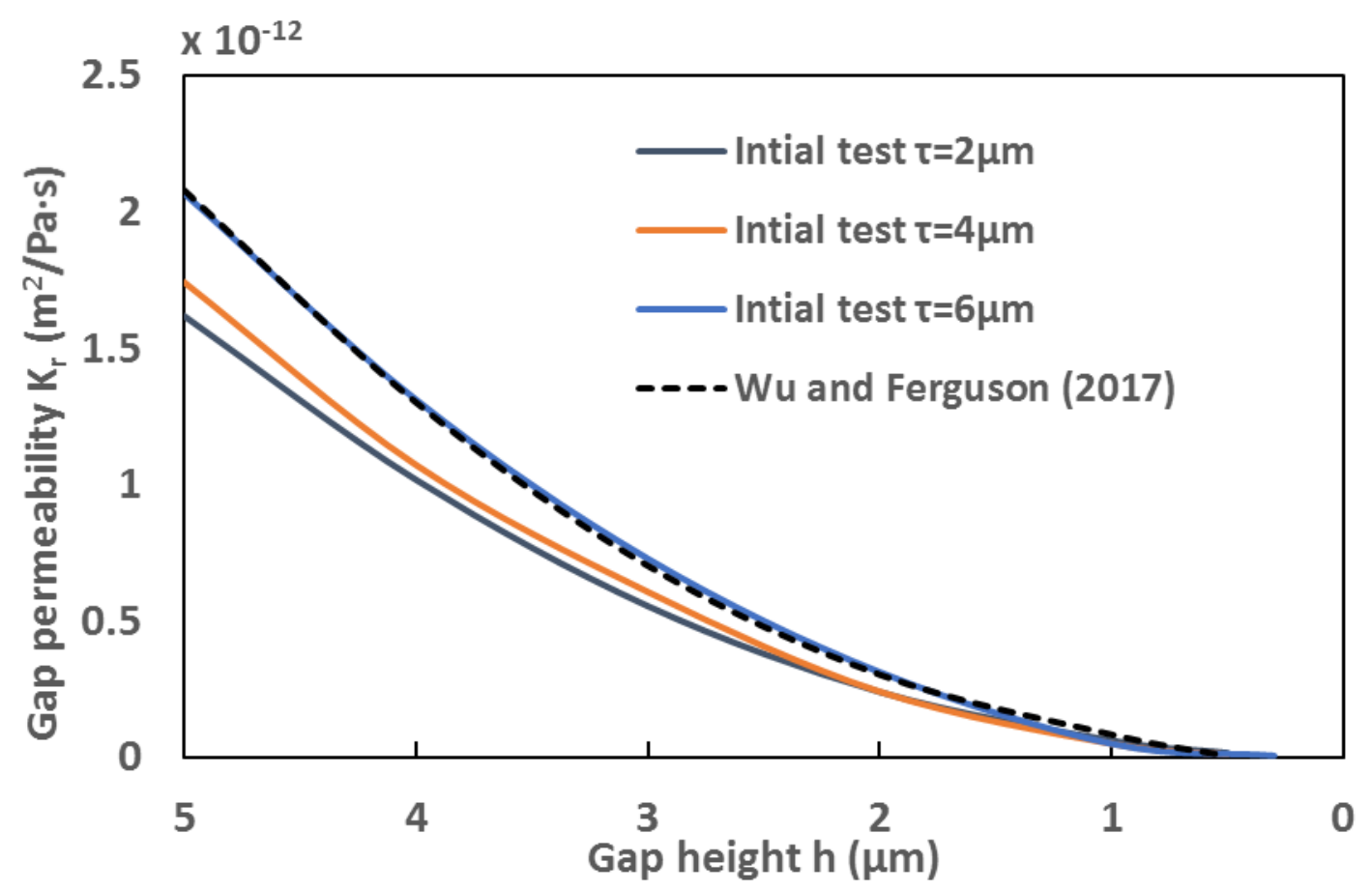

Fig.6 - Model verification against Wu and Ferguson (2017).

494

495

496

497

498

499

500

501

502

503

504

505

506

507

\subsection{Fluid viscosity}

As stated previously, viscosity is a function shear rate and so will depend on both the gap height and the fluid velocity. Since the fluid velocity is driven by the fluid pressure gradient $\Delta p / \Delta r$, viscosity is also dependent on the fluid pressure gradient, such that it can be expressed as $\mu=\mu(h, \Delta p / \Delta r)$. The apparent viscosity as a function of the pressure gradient and gap height is shown in Fig.7 in log-log scale, in which the dashlines are extrapolations to the infinite-shear viscosity $\mu_{\infty}=$ $0.01 \mathrm{~Pa} \cdot \mathrm{s}$. The viscosity charts are plotted by the mean values of the 10 surface samples generated previously, and the error bars are the standard deviations. Incremental convergence tests for the standard deviations were performed for the most divergent case $(h=1 \mu \mathrm{m})$ at all pressure gradients, and the results show that the standard deviations at all pressure gradients decrease with increasing surface sample size and gradually become constant. When the sample sizes are increased 
to 10 , the differences of the standard deviation of the viscosity at all pressure gradients compared to 9 samples are reduced to $5 \%$. Therefore, in this study 10 samples are sufficient to satisfy statistical requirements.

As shown, for a fixed pressure gradient, the fluid viscosity increases as the gap narrows. For example, under the same pressure gradient of $10^{7} \mathrm{~Pa} / \mathrm{m}$, viscosities for the gap height of 16 and $12 \mu \mathrm{m}$ have already reached the infinite-shear viscosity $\mu_{\infty}=0.01 \mathrm{~Pa} \cdot \mathrm{s}$, while the viscosity for $h=4 \mu \mathrm{m}$ is 100 times higher (i.e., $1 \mathrm{~Pa} \cdot \mathrm{s}$ ). Furthermore, the viscosity decreases with increasing fluid pressure gradient (or fluid velocity). For example, by increasing the inlet pressure by 1000 times from the shear thinning threshold, the viscosities of gap heights of $4 \mu \mathrm{m}$ would reduce from the maximum $\mu_{0}$ to the minimum $\mu_{\infty}$. The results demonstrate that the effect of a shear rate dependent viscosity potentially plays a significant role in fluid flow through the gap and so is joint lubrication.

Using a constant viscosity of $1 \mathrm{~Pa} \cdot \mathrm{s}$ or $0.6 \mathrm{~Pa} \cdot \mathrm{s}$ may reflect the general fluid behaviour in the gap, but it fails to capture the synovial fluid behaviour during the joint contact as a process. For a single load event, such as standing up, after a quick squeeze-film stage, the rough surfaces of the upper and lower cartilages will come into contact. When the contact initiates, the total applied load $\sigma_{\mathrm{t}}$ (i.e., body weight) is initially resisted by the fluid pressure $p$ in the gap only. In this instance, the initial viscosity of synovial fluid is expected to be high, as it takes some time for the long molecules in the fluid to re-orientate. Once the relaxation time passes, the synovial fluid begins to thin and eventually reaches a steady state similar to the viscometer experiments. Now in an early contact stage, the majority of the load is taken up by the fluid and hence the fluid pressure gradient is high, the viscosity of the fluid is low and may be close to the infinite shear viscosity. As a result, the fluid 
exits the gap quickly while it closes. With the closure of the gap, the surface asperities start to consolidate and the contact stress $\sigma_{\mathrm{c}}$ will rise and take up the total applied load as per Eq. (14),

$$
\sigma_{t}=\sigma_{c}+p
$$

As the asperity consolidation continues, both the gap height and the fluid pressure gradient will reduce, the viscosity of the remaining synovial fluid will increase as per Fig.7. Therefore, the fluid flow process in temporal cartilage contact is complex, and this complexity cannot be accurately captured by using a constant viscosity value.

\subsection{Gap permeability}

The gap permeability $K_{r}$ was computed by Eq. (4) and plotted in Fig.8 using a loglog scale. The similar convergence study using $h=1 \mu \mathrm{m}$ case as example was carried out to determine the sample size required to estimate the $K_{\mathrm{r}}$. The results also show that 10 samples of rough random surfaces are sufficient to satisfy statistical requirements (less than $5 \%$ change in standard deviation).

$K_{\mathrm{r}}$ is also a function of gap height and fluid pressure gradient because $K_{\mathrm{r}}=$ $\kappa(h) / \mu(h, \Delta p / \Delta r)$. As expected, gap permeability reduces with gap closure due to the reduction in pore space. It is also apparent that gap permeability is very sensitive to the fluid pressure gradient, e.g. at gap height of $8 \mu \mathrm{m}$, the 10 times decrease in pressure gradient (from $10^{7}$ to $10^{6} \mathrm{~Pa} / \mathrm{m}$ ) could lead to a 600 fold decrease in gap permeability.

The gap fluid flow velocity can be found by multiplying the pressure gradient in $\mathrm{X}$-axis to the corresponding gap permeability values in Y-axis (as per Eq. (1)). Moghadam et al. (2014) developed a numerical model to compute the synovial fluid flow between two cartilage surfaces under sinusoidal deformation (at $15 \mathrm{~Hz}$ and 
$15 \%$ strain amplitude). In their study the viscosity was kept constant at 0.6 Pa.s.

They predicted that the maximum flow velocity between two contacting cartilages was around 120-180 $\mu \mathrm{m} / \mathrm{s}$. To compare results using Fig.7 and Fig.8, if we assume that the $15 \%$ cartilage strain in their study corresponds approximately to $15 \%$ roughness strain in the present study (i.e., $h=14 \mu \mathrm{m}$, take an interpolation between $h=16$ and $12 \mu \mathrm{m}$ curves). The reading of pressure gradient corresponding to 0.6 Pa.s from Fig.7 (i.e., around $1.2 \times 10^{6} \mathrm{~Pa} / \mathrm{m}$ ), multiplied by the corresponding gap permeability from Fig. 8 (i.e., around $1.7 \times 10^{-11} \mathrm{~m}^{2} / \mathrm{Pa} \cdot \mathrm{s}$ ), yields a gap flow velocity around $200 \mu \mathrm{m} / \mathrm{s}$, which matches reasonably with Moghadam et al. (2014). In addition, another study by Chen et al. (2011) determined the interfacial flow velocity ranged from 26 to $65 \mu \mathrm{m} / \mathrm{s}$, it should be noted that the flow velocity in Chen's study was obtained from the upmost layer of a tissue engineered cartilage hydrogel that formed on top of the cartilage surface, in which the presence of molecules in the gel and the concentration of synovial fluid are expected to provide additional resistance to the flow. However, this study mainly focuses on the fluid flow from mechanical perspective and hence our prediction tends to be 3 to 7 times greater.

Another observation from Fig. 8 is that the error bars (mean + standard derivation) become more and more divergent with gap closure. When contact initiates, $K_{\mathrm{r}}$ values are relatively convergent regardless of different sample topographies, which indicates at this stage $K_{\mathrm{r}}$ is insensitivity to the variation of cartilage surface topography. This is because the formation of the roughness is assumed to follow the Gaussian distribution that the majority (around 68\%) of the asperity heights would distribute in an interval of one standard deviation from the mean (around $h=5 \mu \mathrm{m}$ ). While in the early contact stage (i.e., $h=16$ to $12 \mu \mathrm{m}$ ) only a few highest asperities are connected and so a relatively generous gap still exists between the highest asperities and the majority of 
surface roughness, from which most of fluid can more easily escape. With the contact continues, $K_{r}$ values become more and more sensitive to the surface topography, because the flow heterogeneity become increasingly significant due to gap closure, especially when the positive roughness peaks are almost flattened (i.e., $h=4$ and 1 $\mu \mathrm{m})$, and fluid flow is forced to take the increasingly tortuous paths in the remaining connected negative roughness pore spaces. Therefore, the cartilage surface topography has increasing influences on the gap permeability when the contact gap closes.

Fig. 8 also provides an index to evaluate the gap permeability. The maximum $K_{\mathrm{r}}$ are capped by the corresponding values when the viscosity of each gap height reaches the infinite-shear viscosity limit of $0.01 \mathrm{~Pa} \cdot \mathrm{s}$, as the fluid viscosity is no longer dependent on the pressure gradient. To the knowledge of the authors, currently there is no effective experimental method to measure the gap permeability either in vivo or in vitro. However, the pressure gradient and gap height can be quantified with advanced equipment. The cartilage contact pressure contours have been mapped by some researchers with modified endoprosthesis integrated with transducers (Rushfeldt et al., 1981), Tekscan knee sensor (Marzo and GurskeDePerio, 2009) and most recently with 3D laser scanning (De Santis et al., 2018). As $95 \%$ of the contact loadings is assumed supported by the interstitial fluid, due to fluid pressure continuity, it is reasonable to assume $95 \%$ of cartilage contact pressure is supported by the gap fluid pressure (Wu and Ferguson, 2017). On the other hand, the measurement of gap thickness for stainless steel on glass in calf serum was also made possible with a commercial optical interferometric tribometer (Myant and Cann, 2013). Therefore, with experimental devices existing for in principle 
measurement of quantified fluid pressure gradient and gap thickness, gap permeability may be evaluated indirectly in the future using Fig. 8 .

Several recent studies have investigated the formulations of cartilage contact during mixed mode lubrication. For example, Sokoloff (2012) modelled the fluid flow in the contact gap by Darcy's law, and hence the gap permeability is required as a key input parameter. Thus, the current study could lead to a more realistic gap permeability estimation. In addition, the formulations of cartilage mixed mode contact are complex because a more complete model should consider the asperity contact and fluid exchange between interstitial fluid in cartilage tissue driven by the pressure difference between the gap and cartilage asperity tissue, rather than assumed equilibrium conditions. A more realistic model would also consider the fluid connection to the underlying cartilage tissue. We plan to extend the gap model to include the underlying cartilage tissue in a future study. This study numerically predicts the gap permeability against a range of gap sizes and fluid pressure gradients in the gap. The outcomes of this study could potentially contribute to the development of more realistic models in the future. A future study will connect this gap with a cartilage bulk deformation model, which should result in a model that is closer to "real world behaviour" occurring in synovial joints.

In summary, this study adopts a displacement-based approach to simulate synovial fluid flow in the contact gap during the gap closure. The purpose of this study is mainly to investigate the non-Newtonian fluid behaviour in the contact gap between two cartilages, under different fluid pressure gradients (representing the fluid pressure in the gap resulting from different load conditions and stages) and gap sizes (i.e., asperity level deformation). The results suggest that the contact gap flow is governed by a combined effect of surface roughness, synovial fluid properties and fluid pressure 
gradient. The presence of roughness in the gap not only changes the intrinsic

632

633

permeability (i.e., the pore spaces in the gap) as per Wu and Ferguson (2017), in a more realistic physiological condition (i.e., non-Newtonian behaviour of synovial fluid), under the change of fluid pressure in the contact gap, cartilage surface roughness could also increase fluid local shearing which can alter the viscosity of synovial fluid, and thereby influence the gap permeability.

\subsection{Discussion on joint lubrication}

The permeability values of cartilage and the gap can shed some light on the plausibility of joint lubrication theories in the mixed mode regime. The bulk permeability of cartilage, dependent on the concentration of aggrecan, is normally in the range of $10^{-15}-10^{-16} \mathrm{~m}^{2} / \mathrm{Pa} \cdot \mathrm{s}$ (Miramini et al., 2017). The permeability at the top surface as a collagen dominant area would be expected to be less than the average permeability, but the increasing actual concentration of aggrecan in a cartilage consolidation process will also reduce the initial cartilage permeability. With this understanding, it is reasonable to take $10^{-15} \mathrm{~m}^{2} / \mathrm{Pa} \cdot \mathrm{s}$ as a reference for comparison.

The results in Fig. 8 show that when the gap height reduces to less than $1 \mu \mathrm{m}$ and fluid pressure gradient reduces below $5 \times 10^{5} \mathrm{~Pa} / \mathrm{m}$, the gap permeability would drop to the order of $10^{-15} \mathrm{~m}^{2} / \mathrm{Pa} \cdot \mathrm{s}$. Therefore, we may tentatively define the following lubrication regimes: (1) from the initial contact to the positive roughness (peaks) being completely flattened (i.e., $h=0$ ) as the mixed mode lubrication regime; (2) $h=0$ to be the onset of boundary only lubrication regime (end of the mixed mode); (3) in the mixed mode regime, when gap permeability reduces to the order of $10^{-15}$ $\mathrm{m}^{2} / \mathrm{Pa} \cdot \mathrm{s}$, that is the same as cartilage tissue, weeping lubrication probably shifts to boosted lubrication, then a more clear picture of the lubrication process during contact begins to emerge. Weeping is likely to be the primary lubrication 
mechanism in mixed mode, as for most of the gap closure the gap permeability is much greater than that of the underlying cartilage. This will cause the gap fluid to exit laterally into the capsule. With contact area increasing, connected porosity decreases to such an extent that boosted lubrication is likely to become dominant over weeping lubrication at some point, probably around the point when permeability of the gap is approximately equal to the cartilage tissue (e.g. when $h$ $=1 \mu \mathrm{m}$ and pressure gradient reduces to less than $5 \times 10^{5} \mathrm{~Pa} / \mathrm{m}$ in this study). The boosted lubrication tends to dominate at the end of mixed mode regime, and it leaves a layer of concentrated protein gel as boundary lubricant that aids boundary lubrication. Therefore, this boosted lubrication regime extends the duration of the mixed mode lubrication regime, further delaying the onset of the boundary (only) lubrication regime. At this turning point when permeability of the gap is equal to the cartilage tissue (i.e., $h=1 \mu \mathrm{m}$ and pressure gradient reduces to less than $5 \times 10^{5}$ $\mathrm{Pa} / \mathrm{m}$ in this study), it can be defined that the gap is functionally closed.

In addition, as shown in Fig.5 (b), when the gap height reduces to zero, the fluid trapped in the valleys of the roughness become isolated, disconnected pools with an average volume of $950940 \mu \mathrm{m}^{3}$ (out of the 10 samples), which is around $13 \%$ of the volume for the initial gap at first contact. This means that around about $13 \%$ of the total fluid in the gap might experience ultrafiltration (boosted lubrication), while around $87 \%$ of the fluid in the gap would be extruded along the gap to the capsule (weeping lubrication).

It should be mentioned that the proposed definition above on gap functional closure is a local criterion. The uneven distribution of interstitial fluid could generate nonuniform cartilage deformation and different gap sizes in different locations, which implies that the fluid exchange (weeping and boosted) in the gap is spatially and locally 
variable. In other words, it is possible for different parts of the gap to be in different lubrication modes. Limitations of this study should be emphasised. Additional to relative permeability, relative path length may also influence the fluid flow from the global perspective. However, the global effect of the relative path length during joint contact cannot be captured in this study because the current gap model is only suitable for investigating the micro-level gap flow. To consider the path length and fully unlock the mystery of fluid flow between cartilage and contact gap, a global and integrated cartilage contact model involving fluid flow in the contact gap, asperity level deformation and cartilage tissue biphasic behaviour needs to be developed. This study represents the first step towards the formulation of a comprehensive cartilage contact model, and clearly further investigations are required.

In addition (and similar to the study of Wu and Ferguson (2017)), this study neglects the bulk deformation of cartilage tissue as a first estimate of the contact gap behaviour. However, role of the bulk deformation of cartilage tissue is clearly required to form an integrated cartilage contact model. When under compression, the cartilage tissue will deform globally, contact drainage paths may lengthen and cartilage may consolidate non-uniformly. For example, in gap sizes could be different between the cartilage perimeter edges where interstitial fluid can more easily exudate compared to the "central area" of the cartilage where the drain paths are longer. In other words, the geometry of the contact gap and the geometry of the cartilage tissue continuously change, and this is not reflected in the current model. Some of these issues can be addressed in future modelling studies, but further experimental work is also required. For example, there is currently no experimental confirmation of how 

and the cartilage bulk deformation.

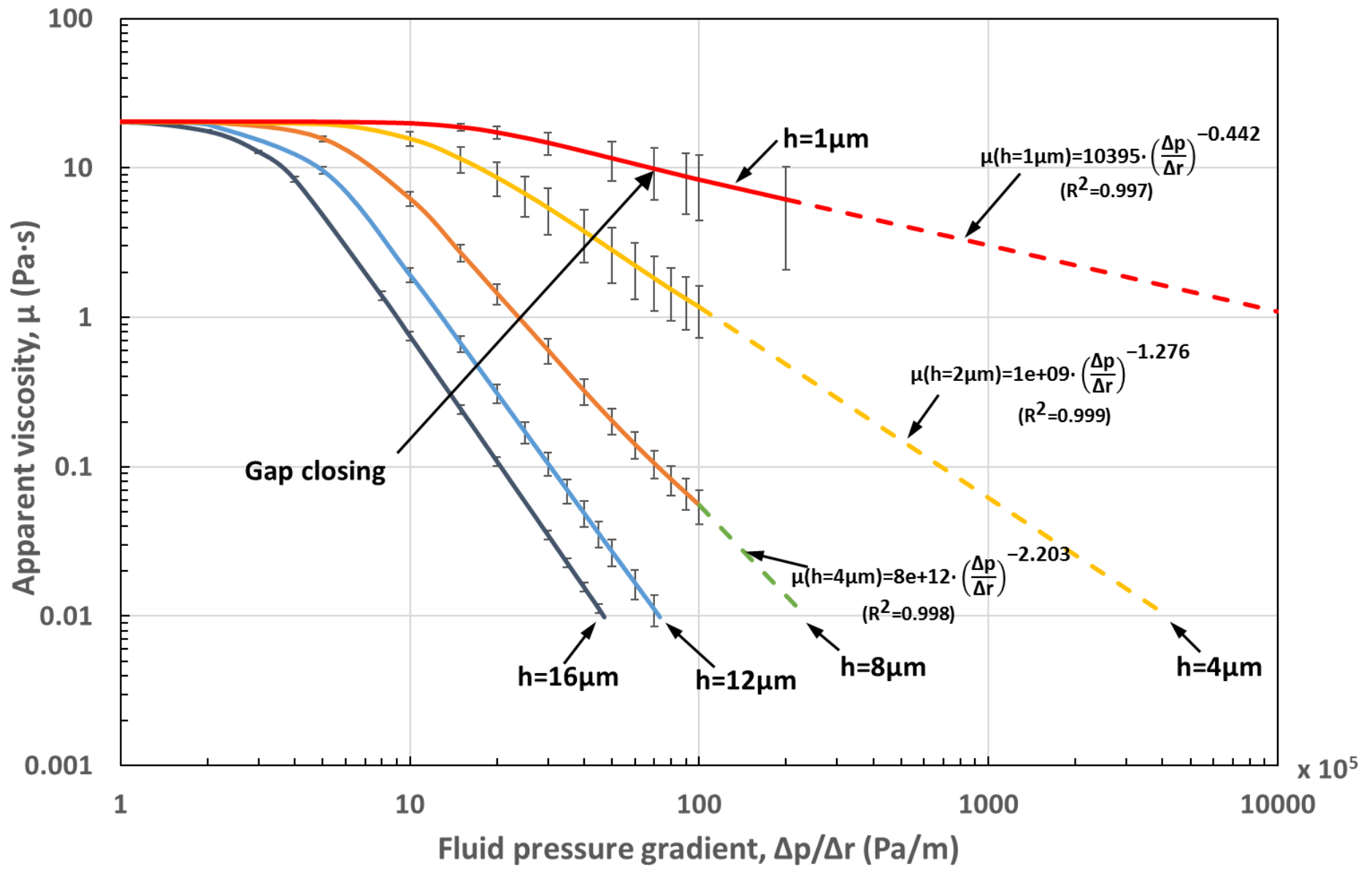

Fig.7 - Apparent viscosity at various gap heights and fluid pressure gradients 


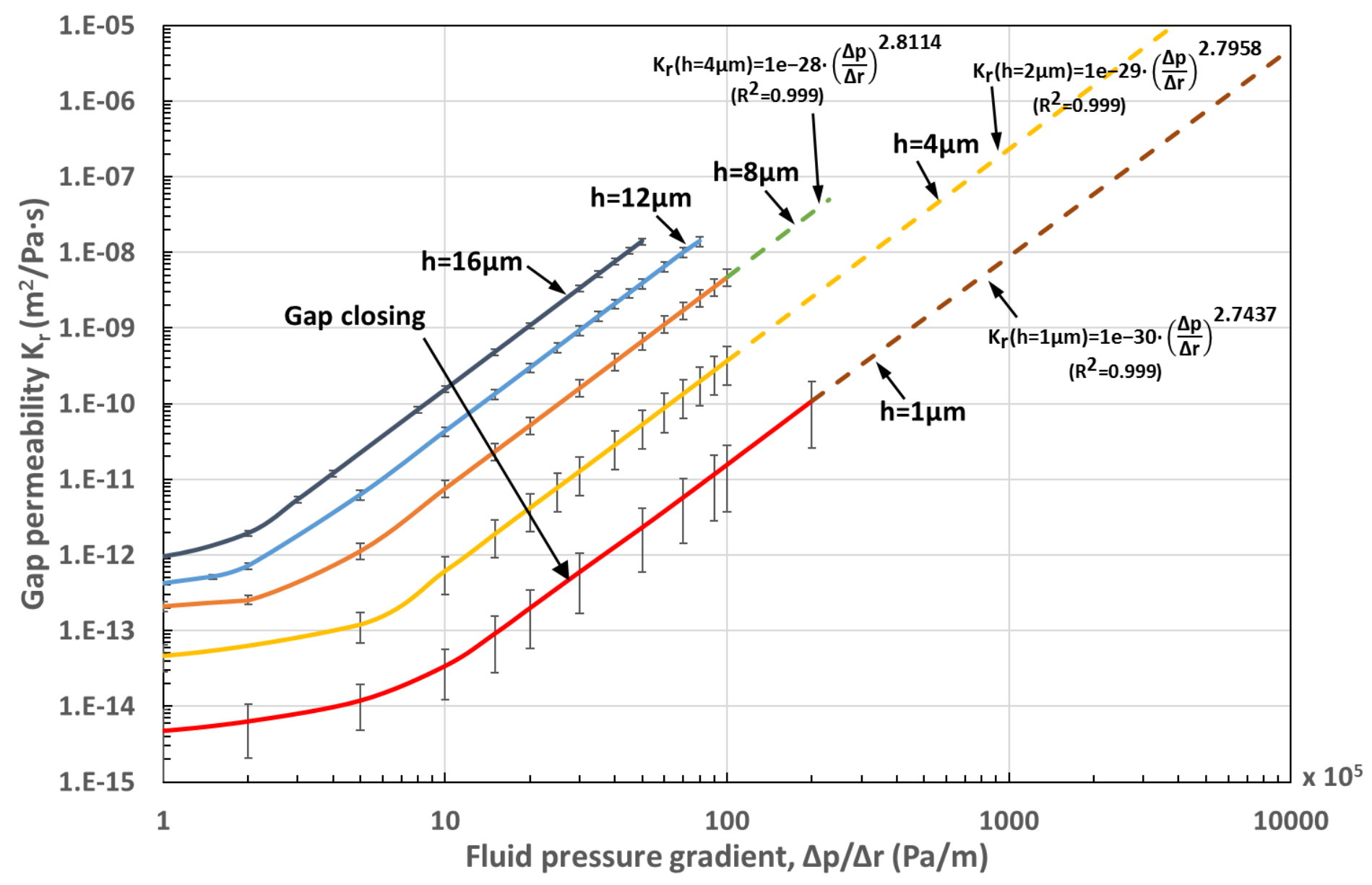

Fig.8 - Gap permeability at various gap heights and fluid pressure gradients 


\section{CONCLUSIONS}

This study investigates the synovial fluid flow behaviour (i.e. permeability) in various sizes of cartilage contract gap by developing CFD models and synthesising cartilage surface with random roughness based on experimental measurements. The CFD model is validated against the study of Wu and Ferguson (2017) and the flow velocity results are consistent with other research findings (Moghadam et al., 2014). The findings are summarised as follows:

- The experimental measurements of bovine media tibia cartilage and surface analysis results show that the averaged roughness $R_{\mathrm{a}}$ is $1.97 \mu \mathrm{m}$ and RMS roughness $R_{\mathrm{q}}$ is $2.44 \mu \mathrm{m}$. The correlation lengths of the secondary and tertiary undulations are round $100 \mu \mathrm{m}$ and $20 \mu \mathrm{m}$, respectively.

- The gap permeability decreases as gap is closing. For example, at pressure gradient of $10^{6} \mathrm{~Pa} / \mathrm{m}$, the decrease of gap height from $4 \mu \mathrm{m}$ to $1 \mu \mathrm{m}$ could result in 20 fold decrease in permeability. In addition, it shows that the gap permeability is very sensitive to the fluid pressure gradient, e.g. at gap height of $8 \mu \mathrm{m}$, the 10 fold decrease in pressure gradient (from $10^{7}$ to $10^{6} \mathrm{~Pa} / \mathrm{m}$ ) could lead to 600 fold decrease in gap permeability. The gap permeability charts developed in this study can be used to estimate the gap permeability under different gap heights and fluid pressure gradients.

- As the cartilage contact gap closes, the permeability of the contact gap gradually approaches that of the cartilage tissue. At some point, e.g., gap height is around $1 \mu \mathrm{m}$ and fluid pressure gradient is below $5 \times 10^{5} \mathrm{~Pa} / \mathrm{m}$, the contact gap may be defined as being "functional closed", as the gap permeability becomes approximately equal to the permeability of the 
cartilage tissue. This suggests that weeping lubrication is the primary mode when gap height and fluid pressure when the contact gap is functionally open, and boosted lubrication becomes significant when the contact gap is functionally closed.

752

753

Acknowledgements

754 The authors acknowledge the funding support from The Australian Research 755 Council (DP180100915) and The University of Melbourne. 


\section{REFERENCES}

Ateshian, G.A., 2009. The role of interstitial fluid pressurization in articular cartilage lubrication. J Biomech 42, 1163-1176.

Barnes, R., 2003. Variogram tutorial. Golden Software.

Bear, J., 1988. Dynamics of fluid in porous media. Dover Publication, New York.

Bergstrom, D., Powell, J., Kaplan, A.F.H., 2007. A ray-tracing analysis of the absorption of light by smooth and rough metal surfaces. J. Appl. Phys. 101, 113504. https://doi.org/10.1063/1.2738417.

Bergstrom, D., Powell, J., Kaplan, A.F.H., 2008. The absorption of light by rough metal surfaces - A three-dimensional ray-tracing analysis. J. Appl. Phys. 103, 103515. https://doi.org/10.1063/1.2930808.

Bhuanantanondh, P., 2009. Rheology of synovial fluid with and without viscosupplements in patients with osteoarthritis: A pilot study. Mater Degree Thesis. The University of British Columbia.

Bujurke, N.M., Kudenatti, R.B., 2006. Surface roughness effects on squeeze film poroelastic bearings. Appl. Math. Comput. 174, 1181-1195.

Chen, T., Buckley, M., Cohen, I., Bonassar, L., Awad, H.A., 2012. Insights into interstitial flow, shear stress, and mass transport effects on ECM heterogeneity in bioreactor-cultivated engineered cartilage hydrogels. Biomech. Model. Mechanobiol. 11, 689-702.

De Santis, R., Gloria, A., Viglione, S., Maietta, S., Nappi, F., Ambrosio, L., Ronca, D., 2018. 3D laser scanning in conjunction with surface texturing to evaluate shift and reduction of the tibiofemoral contact area after meniscectomy. J. Mech. Behav. Biomed. Mater. $88,41-47$.

Fam, H., Bryant, J.T., Kontopoulou, M., 2007. Rheological properties of synovial fluids. Biorheology. 44, 59-74.

Graham, B.T., Moore, A.C., Burris, D.L., Price, C., 2017. Sliding enhances fluid and solute transport into buried articular cartilage contacts. Osteoarthr. Cartilage 25, 2100-2107.

Graindorge, S., Ferrandez, W., Ingham, E., Jin, Z., Twigg, P., Fisher, J., 2006. The role of the surface amorphous layer of articular cartilage in joint lubrication. Proc. Inst. Mech. Eng. H. 220, 597-607.

Greene, G.W., Olszewska, A., Osterberg, M., Zhu, H., Horn, R., 2014. A cartilage-inspired lubrication system. Soft Matter 10, 374-382.

Hu, Y.Z., Tonder, K., 1992. Simulation of 3-D Random Rough-Surface by 2-D Digital-Filter and Fourier-Analysis. Int. J. Mach. Tool. Manu. 32, 83-90.

Lai, W.M., Kuei, S.C., Mow, V.C., 1978. Rheological Equations for Synovial-Fluids. J. Biomech. Eng. 100, 169-186.

Lee, S.S., Duong, C.T., Park, S.H., Cho, Y., Park, S., Park, S., 2013. Frictional response of normal and osteoarthritic articular cartilage in human femoral head. Proc. Inst. Mech. Eng. H 227, 129-137.

Longmore, R.B., GARDNER, D.L., 1975. Development with age of human articular cartilage surface structure. A survey by interference microscopy of the lateral femoral condyle. Ann. Rheum. Dis. 34, 26-37. 
Marzo, J.M., Gurske-DePerio, J., 2009. Effects of medial meniscus posterior horn avulsion and repair on tibiofemoral contact area and peak contact pressure with clinical implication. Am. J. Sports. Med. 37(1), 124-129.

McCutchen, C.W., 1959. Mechanism of Animal Joints: Sponge-hydrostatic and Weeping Bearings. Nature. 184, 1284.

McCutchen, C.W., 1962. The frictional properties of animal joint. Wear. 5, 1-17.

Merkher, Y., Sivan, S., Maroudas, A., Haiperin, G., Yosef, A., 2006. A rational human joint friction test using a human cartilage-on-cartilage arrangemen. Tribol. Lett. 22(1), 2936.

Miramini, S., Smith, D.W., Zhang, L.H., Gardiner, B.S., 2017. The spatio-temporal mechanical environment of healthy and injured human cartilage during sustained activity and its role in cartilage damage. J. Mech. Behav. Biomed. 74, 1-10.

Moghadam, M.N., Abdel-Sayed, P., Camine, V.M., Pioletti, D.P., 2015. Impact of synovial fluid flow on temperature regulation in knee cartilage. J. Biomech. 48, 370-374.

Moore, A.C., Burris, D.L., 2017. Tribological rehydration of cartilage and its potential role in preserving joint health. Osteoarthr. Cartilage 25, 99-107.

Mow, V.C., Ateshian, G.A., Spilker, R.L., 1993. Biomechanics of diarthrodial joints: a review of twenty years of progress. J. Biomech. Eng. 115, 460-467.

Myant, C., Cann, P., 2013. In contact observation of model synovial fluid lubricating mechanisms. Tribol. Int. 63, 97-104.

Neu, C.P., Komvopoulos, K., Reddi, A.H., 2008. The interface of functional biotribology and regenerative medicine in synovial joints. Tissue Eng. Part B Rev. 14(3), 235-247.

Rushfeldt, P.D., Mann, R.W., Harris, W.H., 1981. Improved techniques for measuring in vitro the geometry and pressure distribution in the human acetabulum. II Instrumented endoprosthesis measurement of articular surface pressure distribution. J. Biomech. 14, 315-323.

Schurz, J., Ribitsch, V., 1987. Rheology of Synovial-Fluid. Biorheology. 24, 385-399.

Smyth, P.A., Rifkin, R.E., Jackson, R.L., Hanson, R.R., 2012. A Surface Roughness Comparison of Cartilage in Different Types of Synovial Joints. J. Biomech. Eng. 134, 021006. DOI: $10.1115 / 1.4005934$.

Shekhawat, V.K., Laurent, M.P., Muehleman, C., Wimmer, M.A., 2009. Surface topography of viable articular cartilage measured with scanning white light interferometry. Osteoarthritis. Cartilage 17, 1197-1203.

Sokoloff, J.B., 2012. Theory of the effects of surface roughness on fluid lubrication of hydrogels. Soft Matter 8, 8164-8170.

Walker, P.S., Dowson, D., Longfield, M.D., Wright, V., 1968. Boosted Lubrication in Synovial Joints by Fluid Entrapment and Enrichment. Ann. Rheum. Dis. 27, 512-520.

Walker, P.S., Dowson, D., Longfield, M.D., Wright, V., 1969. Lubrication of human joints. Ann. Rheum. Dis. 28, 194.

Wu, J.J., 2000. Simulation of rough surfaces with FFT. Tribol. Int. 33, 47-58.

Wu, Y.B., Ferguson, S.J., 2017. The influence of cartilage surface topography on fluid flow in the intra-articular gap. Comput. Methods Biomec. 20, 250-259. 
Zhang, L.H., Miramini, S., Smith, D.W., Gardiner, B.S., Grodzinsky, A.J., 2015. Time Evolution of Deformation in a Human Cartilage Under Cyclic Loading. Ann. Biomed. Eng. 43, 1166-1177. 


\section{University Library}

\section{- M M N E R VA A gateway to Melbourne's research publications}

Minerva Access is the Institutional Repository of The University of Melbourne

Author/s:

Liao, J;Smith, DW;Miramini, S;Thibbotuwawa, N;Gardiner, BS;Zhang, L

Title:

The investigation of fluid flow in cartilage contact gap

Date:

2019-07-01

Citation:

Liao, J., Smith, D. W., Miramini, S., Thibbotuwawa, N., Gardiner, B. S. \& Zhang, L. (2019). The investigation of fluid flow in cartilage contact gap. JOURNAL OF THE MECHANICAL BEHAVIOR OF BIOMEDICAL MATERIALS, 95, pp.153-164. https://doi.org/10.1016/ j.jmbbm.2019.04.008.

Persistent Link:

http://hdl.handle.net/11343/245473 